\title{
Probing non-standard interactions at Daya Bay
}

\author{
Sanjib Kumar Agarwalla, ${ }^{a}$ Partha Bagchi, ${ }^{a}$ David V. Forero ${ }^{b, c}$ and Mariam Tórtola ${ }^{b}$ \\ ${ }^{a}$ Institute of Physics, Sachivalaya Marg, \\ Sainik School Post, Bhubaneswar 751005, India \\ ${ }^{b}$ AHEP Group, Institut de Física Corpuscular - C.S.I.C./Universitat de València, \\ Parc Cientific de Paterna, C/ Catedratico José Beltrán, 2 E-46980 Paterna (València), Spain \\ ${ }^{c}$ Center for Neutrino Physics, Virginia Tech, \\ Blacksburg, VA 24061, U.S.A. \\ E-mail: sanjib@iopb.res.in, partha@iopb.res.in, dvanegas@ific.uv.es, \\ mariam@ific.uv.es
}

ABSTRACT: In this article we consider the presence of neutrino non-standard interactions (NSI) in the production and detection processes of reactor antineutrinos at the Daya Bay experiment. We report for the first time, the new constraints on the flavor non-universal and flavor universal charged-current NSI parameters, estimated using the currently released 621 days of Daya Bay data. New limits are placed assuming that the new physics effects are just inverse of each other in the production and detection processes. With this special choice of the NSI parameters, we observe a shift in the oscillation amplitude without distorting the $L / E$ pattern of the oscillation probability. This shift in the depth of the oscillation dip can be caused by the NSI parameters as well as by $\theta_{13}$, making it quite difficult to disentangle the NSI effects from the standard oscillations. We explore the correlations between the NSI parameters and $\theta_{13}$ that may lead to significant deviations in the reported value of the reactor mixing angle with the help of iso-probability surface plots. Finally, we present the limits on electron, muon/tau, and flavor universal (FU) NSI couplings with and without considering the uncertainty in the normalization of the total event rates. Assuming a perfect knowledge of the event rates normalization, we find strong upper bounds $\sim 0.1 \%$ for the electron and FU cases improving the present limits by one order of magnitude. However, for a conservative error of $5 \%$ in the total normalization, these constraints are relaxed by almost one order of magnitude.

Keywords: Neutrino Physics, Beyond Standard Model

ARXIV EPRINT: 1412.1064 


\section{Contents}

1 Introduction 1

2 Implementing NSI in modern reactor experiments $\quad 4$

2.1 Effective antineutrino survival probability in reactor experiments 5

2.2 Special case of NSI: $\varepsilon_{e \gamma}^{s}=\varepsilon_{\gamma e}^{d *} \quad 6$

2.3 Impact of the NSI parameters on the effective probability 10

2.4 Correlations between NSI parameters and $\theta_{13}$ : iso-probability plots 11

3 Data analysis of modern reactor experiments $\quad 13$

4 Bounds on NSI from Daya Bay without normalization error $\quad \mathbf{1 5}$

$\begin{array}{lll}4.1 \text { Constraints on electron-NSI couplings } & 15\end{array}$

$\begin{array}{lll}4.2 & \text { Constraints on muon/tau-NSI couplings } & 16\end{array}$

$\begin{array}{lll}4.3 & \text { Constraints for the flavor-universal NSI case } & 18\end{array}$

5 Bounds on NSI from Daya Bay with 5\% normalization error $\quad 20$

6 Comparing NSI constraints from 217 and 621 days of Daya Bay run $\quad 22$

$\begin{array}{lll}7 & \text { Summary and conclusions } & 25\end{array}$

A Effective survival probability with the NSI parameters: $\varepsilon_{e \gamma}^{s} \neq \varepsilon_{\gamma e}^{d *} \quad 27$

A.1 Presence of the NSI parameters only at the production stage 27

A.2 NSI at the source and detector with the same magnitude and different phases 27

\section{Introduction}

The recent discovery of the smallest neutrino mixing angle $\theta_{13}$ by the modern reactor antineutrino experiments Daya Bay [1-3] and RENO [4] has firmly established the three-flavor neutrino paradigm [5-7] and signifies an important development towards our understanding of the structure of the neutrino mass matrix, whose precise reconstruction would shed light on the underlying new physics that gives rise to neutrino mass and mixing [8-10]. Another reactor electron antineutrino disappearance experiment: Double Chooz [11, 12], and the two accelerator electron (anti-)neutrino appearance experiments: MINOS [13] (completed) and T2K $[14,15]$ (presently running) have also confirmed the non-zero and moderately large value of $\theta_{13}$ in the standard three-flavor oscillation scenario. It is quite remarkable to see that with 621 days of data taking and using the merit of identical multi-detector setup, the Daya Bay experiment reveals a non-zero value of $\theta_{13}$ at more than $16 \sigma$ and suggests a best-fit value of $\sin ^{2} 2 \theta_{13}=0.084 \pm 0.005$ [3]. This data provides a relative $1 \sigma$ 
precision of $6 \%$ on $\sin ^{2} 2 \theta_{13}$ which is already better than the precision achieved on $\sin ^{2} \theta_{23}$. Undoubtedly, this high precision measurement of the 1-3 mixing angle has speeded up the search for the neutrino mass ordering and the possible presence of a CP-violating phase in current and future neutrino oscillation experiments [16-20].

To explain the presence of small neutrino masses and relatively large neutrino mixings as indicated by neutrino oscillation data, various neutrino mass models have been proposed. These neutrino mass models come in various categories such as the cases where neutrinos acquire mass via the popular seesaw mechanism [21-31]. We find also models where neutrinos get mass radiatively due to the presence of extra Higgs bosons [32-34] or low energy supersymmetric hybrid models with spontaneous or bilinear breaking of R-parity [35, 36]. The structure of the standard electroweak neutral and charged currents gets affected by the presence of these mechanisms responsible for the neutrino mass generation [25]. In most of the cases, in the low energy regime, these effects are known as non-standard interactions (NSI). Various extensions of the Standard Model (SM), such as left-right symmetric models and supersymmetric models with R-parity violation, predict NSI of neutrinos with other fermions [37-45]. The NSI in these models are usually generated via the exchange of new massive particles at low energies.

Neutrino NSI may be of charged-current (CC) or neutral-current (NC) type, and they can be classified in two main categories: flavor-changing NSI, when the flavor of the leptonic current involved in the process is changed, or flavor-conserving non-universal NSI, when the lepton flavor is not changed in the process but the strength of the interaction depends on it, violating the weak universality. In the low energy regime, these new interactions may be parameterized in the form of effective four-fermion Lagrangians:

$$
\begin{aligned}
\mathcal{L}_{\mathrm{CC}-\mathrm{NSI}} & =\frac{G_{F}}{\sqrt{2}} \sum_{f, f^{\prime}} \varepsilon_{\alpha \beta}^{s d, f f^{\prime}}\left[\bar{\nu}_{\beta} \gamma^{\rho}\left(1-\gamma^{5}\right) \ell_{\alpha}\right]\left[\bar{f}^{\prime} \gamma_{\rho}\left(1 \pm \gamma^{5}\right) f\right], \\
\mathcal{L}_{\mathrm{NC}-\mathrm{NSI}} & =\frac{G_{F}}{\sqrt{2}} \sum_{f} \varepsilon_{\alpha \beta}^{m, f f}\left[\bar{\nu}_{\beta} \gamma^{\rho}\left(1-\gamma^{5}\right) \nu_{\alpha}\right]\left[\bar{f} \gamma_{\rho}\left(1 \pm \gamma^{5}\right) f\right] .
\end{aligned}
$$

where $G_{F}$ is the Fermi constant, $\alpha$ and $\beta$ are neutrino or lepton flavor indices, $f$ and $f^{\prime}$ label light SM fermions, and the dimensionless coefficients $\varepsilon$ parametrize the strength of the interaction. Here we denote the CC-NSI couplings as $\varepsilon_{\alpha \beta}^{s d, f f^{\prime}}$ since they affect in general the source $(\mathrm{s})$ and detector $(\mathrm{d})$ interactions at neutrino experiments, while $\varepsilon_{\alpha \beta}^{m, f f}$ refers to the NC-NSI couplings generally affecting the neutrino propagation in matter $(\mathrm{m})$. Note that in eqs. (1.1) and (1.2) we have assumed for the new interactions the same Lorentz structure as for the SM weak interactions, $(\mathrm{V} \pm \mathrm{A})$. Even though more general expressions are possible within a generic structure of operators, as shown in ref. [46], these are the dominant contributions for reactor experiments, where we will focus our attention in this work.

NSI effects may appear at three different stages in a given neutrino experiment, namely neutrino production, neutrino propagation from the source to the detector, and neutrino detection. In a short-baseline reactor experiment, the effects on the neutrino propagation are negligible since it happens mainly in vacuum. Therefore, the new generation of shortbaseline reactor experiments, such as Daya Bay, offers an excellent scenario to probe the 
presence of NSI at the neutrino production and detection, free of any degeneracy with NSI propagation effects. Actually, some work has already been done in the context of NSI at short-baseline reactor experiments. In particular, forecasts for the sensitivity of Daya Bay to NSI have been published, for instance, in ref. [47]. More recently, another article presented constraints on neutrino NSI using the previous Daya Bay data set [48]. The results derived there are in general agreement with some of the cases we discuss in section 4. Nevertheless, our paper also studies the phenomenology of some other interesting cases not considered in ref. [48], and provides a detailed description of the effect of NSI in the neutrino survival probability. Finally, we also discuss the fragility of the bounds on the NSI couplings derived using Daya Bay data against the presence of an uncertainty on the total event rate normalization in the statistical analysis of reactor data.

Given the production and detection neutrino processes involved in short-baseline reactor neutrino experiments ( $\beta$-decay and inverse $\beta$-decay), the NSI parameters relevant for these experiments are $\varepsilon_{e \alpha}^{u d}$, i.e., the CC-NSI couplings between up and down quarks, positrons and antineutrinos of flavor $\alpha$. In the literature we can find the following $90 \%$ C.L. bounds on these parameters [49]:

$$
\left|\varepsilon_{e \alpha}^{u d, V}\right|<0.041, \quad\left|\varepsilon_{e \mu}^{u d, L}\right|<0.026, \quad\left|\varepsilon_{e \mu}^{u d, R}\right|<0.037,
$$

coming from unitarity constraints on the CKM matrix as well as from the non-observation of neutrino oscillations in the NOMAD experiment. Here the couplings with different chirality are related by: ${ }^{1} \varepsilon_{\alpha \beta}^{f f^{\prime}, V}=\varepsilon_{\alpha \beta}^{f f^{\prime}, L}+\varepsilon_{\alpha \beta}^{f f^{\prime}, R}$. Other constraints on neutrino NSI couplings using solar and reactor neutrinos have been given in [50-55]. On the other hand, NSI have also been studied in the context of laboratory experiments with accelerators in refs. [53, 56-58], while bounds from atmospheric neutrino data have been presented in refs. $[59,60]$. Recently a forecast for the sensitivity to NSI of the future PINGU detector has been presented in [61]. Future medium-baseline reactor experiments like JUNO can also serve as test bed to look for NSI [62].

This paper is organized as follows. In section 2, we describe the procedure for implementing the NSI effect in the modern reactor experiments. There, we derive the effective antineutrino survival probability expressions which we use later to analyze the Daya Bay data. We also give plots to discuss in detail the impact of the NSI parameters on the effective probability considering a special case where $\varepsilon_{e \gamma}^{s}=\varepsilon_{\gamma e}^{d *}$. We also show the possible correlations between the NSI parameters and $\theta_{13}$ with the help of iso-probability surface plots. Section 3 describes the numerical methods adopted to analyze the reactor data. Apart from this, a brief description of the Daya Bay experiment and the important features of its present data set which are relevant for the fit are also given in this section. Section 4 presents the constraints on the NSI parameters imposed by the current Daya Bay data assuming a perfect knowledge of the event rates normalization. Next we derive the bounds on the NSI parameters taking into account the uncertainty in the normalization of event rates with a prior of $5 \%$ in section 5 . In section 6 , we compare the constraints on

\footnotetext{
${ }^{1}$ The NSI parameters probed in our analysis get contributions from the $(\mathrm{V} \pm \mathrm{A})$ operators in eq. (1.1) and therefore they can be generally expressed as $\varepsilon_{\alpha \beta}^{u d, \mathrm{~V} \pm \mathrm{A}}$ or equivalently $\varepsilon_{\alpha \beta}^{u d, \mathrm{~L} \pm \mathrm{R}}$. However, for simplicity, we have dropped the chirality indices all over the paper.
} 
the NSI parameters obtained with the current 621 days of Daya Bay data with the limits derived using the previously released 217 days of Daya Bay data. Finally, we summarize and draw our conclusions in section 7 . In appendix A, we give the effective antineutrino survival probability expressions for the physical situations where $\varepsilon_{e \gamma}^{s} \neq \varepsilon_{\gamma e}^{d *}$.

\section{Implementing NSI in modern reactor experiments}

According to the usual procedure followed in the non-standard analyses of reactor data [47, 63], we start by re-defining the neutrino flavour states in the presence of NSI in the source and detection processes. For the initial (at source) and final (at detector) neutrino flavor states, we have [64-67]:

$$
\left|\nu_{\alpha}^{s}\right\rangle=\frac{1}{N_{\alpha}^{s}}\left(\left|\nu_{\alpha}\right\rangle+\sum_{\gamma} \varepsilon_{\alpha \gamma}^{s}\left|\nu_{\gamma}\right\rangle\right), \quad\left\langle\nu_{\beta}^{d}\right|=\frac{1}{N_{\beta}^{d}}\left(\left\langle\nu_{\beta}\right|+\sum_{\eta} \varepsilon_{\eta \beta}^{d}\left\langle\nu_{\eta}\right|\right),
$$

while the redefinition of the antineutrino flavor states is given by:

$$
\left|\bar{\nu}_{\alpha}^{s}\right\rangle=\frac{1}{N_{\alpha}^{s}}\left(\left|\bar{\nu}_{\alpha}\right\rangle+\sum_{\gamma} \varepsilon_{\alpha \gamma}^{s *}\left|\bar{\nu}_{\gamma}\right\rangle\right), \quad\left\langle\bar{\nu}_{\beta}^{d}\right|=\frac{1}{N_{\beta}^{d}}\left(\left\langle\bar{\nu}_{\beta}\right|+\sum_{\eta} \varepsilon_{\eta \beta}^{d *}\left\langle\bar{\nu}_{\eta}\right|\right) .
$$

The normalization factors required to obtain an orthonormal basis can be expressed as:

$$
N_{\alpha}^{s}=\sqrt{\left[\left(1+\varepsilon^{s}\right)\left(1+\varepsilon^{s \dagger}\right)\right]_{\alpha \alpha}}, \quad N_{\beta}^{d}=\sqrt{\left[\left(1+\varepsilon^{d \dagger}\right)\left(1+\varepsilon^{d}\right)\right]_{\beta \beta}},
$$

and the neutrino mixing between flavor and mass eigenstates is given by the usual expressions:

$$
\left|\nu_{\alpha}\right\rangle=\sum_{k} U_{\alpha k}^{*}\left|\nu_{k}\right\rangle, \quad\left|\bar{\nu}_{\alpha}\right\rangle=\sum_{k} U_{\alpha k}\left|\bar{\nu}_{k}\right\rangle .
$$

The correct normalization of the neutrino states in presence of NSI is a very important point, required to obtain a total neutrino transition probability normalized to 1 . However, one has to consider that when dealing with a non-orthonormal neutrino basis, the normalization of neutrino states will affect not only the neutrino survival probability but also the calculation of the produced neutrino fluxes and detection cross sections. In this case, as shown in ref. [68], all the normalization terms coming from $N_{\alpha}^{s}$ and $N_{\beta}^{d}$ will cancel while convoluting the neutrino oscillation probabilities, cross sections, and neutrino fluxes to estimate the number of events in a given experiment such as Daya Bay. This is due to the fact that the SM cross sections and neutrino fluxes used in our simulation have been theoretically derived assuming an orthonormal neutrino basis and therefore they need to be corrected. Then, from here we can consider the following effective redefinition of neutrino and antineutrino states:

$$
\begin{array}{lll}
\left|\nu_{\alpha}^{s}\right\rangle_{\mathrm{eff}}=\left|\nu_{\alpha}\right\rangle+\sum_{\gamma} \varepsilon_{\alpha \gamma}^{s}\left|\nu_{\gamma}\right\rangle, & \left\langle\left.\nu_{\beta}^{d}\right|_{\mathrm{eff}}=\left\langle\nu_{\beta}\right|+\sum_{\eta} \varepsilon_{\eta \beta}^{d}\left\langle\nu_{\eta}\right|,\right. \\
\left|\bar{\nu}_{\alpha}^{s}\right\rangle_{\mathrm{eff}}=\left|\bar{\nu}_{\alpha}\right\rangle+\sum_{\gamma} \varepsilon_{\alpha \gamma}^{s *}\left|\bar{\nu}_{\gamma}\right\rangle, & \left\langle\left.\bar{\nu}_{\beta}^{d}\right|_{\mathrm{eff}}=\left\langle\bar{\nu}_{\beta}\right|+\sum_{\eta} \varepsilon_{\eta \beta}^{d *}\left\langle\bar{\nu}_{\eta}\right|,\right.
\end{array}
$$


where we have dropped the normalization factors that will cancel in the Monte Carlo simulation of Daya Bay data. From these effective neutrino states we will calculate an effective neutrino oscillation probability, that will be used all along our analysis. Note that when we will discuss the features of the probability prior to the simulation of a particular experiment, we will always refer to the effective probability, that might be greater than one.

\subsection{Effective antineutrino survival probability in reactor experiments}

The effective antineutrino transition probability from flavor $\alpha$ to $\beta$ after traversing a distance $L$ from source to detector is defined as:

$$
P_{\bar{\nu}_{\alpha}^{s} \rightarrow \bar{\nu}_{\beta}^{d}}=\left|\left\langle\bar{\nu}_{\beta}^{d}|\exp (-i H L)| \bar{\nu}_{\alpha}^{s}\right\rangle\right|^{2} .
$$

In terms of the neutrino mass differences and mixing angles, this transition probability in vacuum may be written as:

$$
\begin{aligned}
P_{\bar{\nu}_{\alpha}^{s} \rightarrow \bar{\nu}_{\beta}^{d}}= & \sum_{j, k} Y_{\alpha \beta}^{j} Y_{\alpha \beta}^{k *}-4 \sum_{j>k} \mathcal{R}\left\{Y_{\alpha \beta}^{j} Y_{\alpha \beta}^{k *}\right\} \sin ^{2}\left(\frac{\Delta m_{j k}^{2} L}{4 E}\right) \\
& +2 \sum_{j>k} \mathcal{I}\left\{Y_{\alpha \beta}^{j} Y_{\alpha \beta}^{k *}\right\} \sin \left(\frac{\Delta m_{j k}^{2} L}{2 E}\right),
\end{aligned}
$$

where $\Delta m_{j k}^{2}=m_{j}^{2}-m_{k}^{2}$. In the case of standard oscillations, $Y_{\alpha \beta}^{j}$ is defined as:

$$
Y_{\alpha \beta}^{j} \equiv U_{\beta j}^{*} U_{\alpha j} .
$$

In presence of NSI, however, according to the definition of neutrino states in eq. (2.2), this expression is modified as follows [63]:

$$
Y_{\alpha \beta}^{j} \equiv U_{\beta j}^{*} U_{\alpha j}+\sum_{\gamma} \varepsilon_{\alpha \gamma}^{s *} U_{\beta j}^{*} U_{\gamma j}+\sum_{\eta} \varepsilon_{\eta \beta}^{d *} U_{\eta j}^{*} U_{\alpha j}+\sum_{\gamma, \eta} \varepsilon_{\alpha \gamma}^{s *} \varepsilon_{\eta \beta}^{d *} U_{\eta j}^{*} U_{\gamma j} .
$$

To obtain the $\bar{\nu}_{e}$ survival probability in a reactor experiment, where an electron antineutrino is produced at the source and a positron is detected inside the detector, one has to replace $\alpha$ and $\beta$ by e in eq. (2.8). For the NSI parameters, we adopt the following parametrization by splitting the new couplings into its absolute value and its phase:

$$
\varepsilon_{e \gamma}^{s} \equiv\left|\varepsilon_{e \gamma}^{s}\right| \mathrm{e}^{\mathrm{i} \phi_{e \gamma}^{s}} \quad \text { and } \quad \varepsilon_{\eta e}^{d} \equiv\left|\varepsilon_{\eta e}^{d}\right| \mathrm{e}^{\mathrm{i} \phi_{\eta e}^{d}} .
$$

Now expanding the various terms of the general transition probability as given in eq. (2.8) and using the parametrization above, we obtain the effective $\bar{\nu}_{e}$ survival probability:

$$
\begin{aligned}
P_{\bar{\nu}_{e}^{s} \rightarrow \bar{\nu}_{e}^{d}}= & P_{\bar{\nu}_{e} \rightarrow \bar{\nu}_{e}}^{\mathrm{SM}}+P_{\text {non-osc }}^{\mathrm{NSI}}+P_{\mathrm{osc}-\text { atm }}^{\mathrm{NSI}}+P_{\mathrm{osc}-\text { solar }}^{\mathrm{NSI}} \\
& +\mathcal{O}\left[\varepsilon^{3}, s_{13}^{3}, \varepsilon^{2} s_{13}, \varepsilon s_{13}^{2}, \varepsilon s_{13}\left(\frac{\Delta m_{21}^{2} L}{2 E}\right), \varepsilon\left(\frac{\Delta m_{21}^{2} L}{2 E}\right)^{2}, s_{13}^{2}\left(\frac{\Delta m_{21}^{2} L}{2 E}\right)\right],
\end{aligned}
$$

where the Standard Model (SM) contribution is given by

$$
P_{\bar{\nu}_{e} \rightarrow \bar{\nu}_{e}}^{\mathrm{SM}}=1-\sin ^{2} 2 \theta_{13}\left(c_{12}^{2} \sin ^{2} \Delta_{31}+s_{12}^{2} \sin ^{2} \Delta_{32}\right)-c_{13}^{4} \sin ^{2} 2 \theta_{12} \sin ^{2} \Delta_{21},
$$


with $s_{i j}=\sin \theta_{i j}, c_{i j}=\cos \theta_{i j}$, and $\Delta_{i j}=\Delta m_{i j}^{2} L / 4 E$. The various NSI terms of the effective survival probability in eq. (2.12) can be written as:

$$
\begin{aligned}
P_{\text {non-osc }}^{\mathrm{NSI}}= & 2\left(\left|\varepsilon_{e e}^{d}\right| \cos \phi_{e e}^{d}+\left|\varepsilon_{e e}^{s}\right| \cos \phi_{e e}^{s}\right)+\left|\varepsilon_{e e}^{d}\right|^{2}+\left|\varepsilon_{e e}^{s}\right|^{2}+2\left|\varepsilon_{e e}^{d}\right|\left|\varepsilon_{e e}^{s}\right| \cos \left(\phi_{e e}^{d}-\phi_{e e}^{s}\right)(2.14) \\
& +2\left|\varepsilon_{e e}^{d}\right|\left|\varepsilon_{e e}^{s}\right| \cos \left(\phi_{e e}^{d}+\phi_{e e}^{s}\right)+2\left|\varepsilon_{e \mu}^{s}\right|\left|\varepsilon_{\mu e}^{d}\right| \cos \left(\phi_{e \mu}^{s}+\phi_{\mu e}^{d}\right)+2\left|\varepsilon_{e \tau}^{s}\right|\left|\varepsilon_{\tau e}^{d}\right| \cos \left(\phi_{e \tau}^{s}+\phi_{\tau e}^{d}\right), \\
P_{\mathrm{osc}-\mathrm{atm}}^{\mathrm{NSI}}= & 2\left\{s_{13} s_{23}\left[\left|\varepsilon_{e \mu}^{s}\right| \sin \left(\delta-\phi_{e \mu}^{s}\right)-\left|\varepsilon_{\mu e}^{d}\right| \sin \left(\delta+\phi_{\mu e}^{d}\right)\right]\right. \\
& +s_{13} c_{23}\left[\left|\varepsilon_{e \tau}^{s}\right| \sin \left(\delta-\phi_{e \tau}^{s}\right)-\left|\varepsilon_{\tau e}^{d}\right| \sin \left(\delta+\phi_{\tau e}^{d}\right)\right] \\
& -s_{23} c_{23}\left[\left|\varepsilon_{e \mu}^{s}\right|\left|\varepsilon_{\tau e}^{d}\right| \sin \left(\phi_{e \mu}^{s}+\phi_{\tau e}^{d}\right)+\left|\varepsilon_{e \tau}^{s}\right|\left|\varepsilon_{\mu e}^{d}\right| \sin \left(\phi_{e \tau}^{s}+\phi_{\mu e}^{d}\right)\right] \\
& \left.-c_{23}^{2}\left|\varepsilon_{e \tau}^{s}\right|\left|\varepsilon_{\tau e}^{d}\right| \sin \left(\phi_{e \tau}^{s}+\phi_{\tau e}^{d}\right)-s_{23}^{2}\left|\varepsilon_{e \mu}^{s}\right|\left|\varepsilon_{\mu e}^{d}\right| \sin \left(\phi_{e \mu}^{s}+\phi_{\mu e}^{d}\right)\right\} \sin \left(2 \Delta_{31}\right) \\
& -4\left\{s_{13} s_{23}\left[\left|\varepsilon_{e \mu}^{s}\right| \cos \left(\delta-\phi_{e \mu}^{s}\right)+\left|\varepsilon_{\mu e}^{d}\right| \cos \left(\delta+\phi_{\mu e}^{d}\right)\right]\right. \\
& +s_{13} c_{23}\left[\left|\varepsilon_{e \tau}^{s}\right| \cos \left(\delta-\phi_{e \tau}^{s}\right)+\left|\varepsilon_{\tau e}^{d}\right| \cos \left(\delta+\phi_{\tau e}^{d}\right)\right] \\
& +s_{23} c_{23}\left[\left|\varepsilon_{e \mu}^{s}\right|\left|\varepsilon_{\tau e}^{d}\right| \cos \left(\phi_{e \mu}^{s}+\phi_{\tau e}^{d}\right)+\left|\varepsilon_{e \tau}^{s}\right|\left|\varepsilon_{\mu e}^{d}\right| \cos \left(\phi_{e \tau}^{s}+\phi_{\mu e}^{d}\right)\right] \\
& \left.+c_{23}^{2}\left|\varepsilon_{e \tau}^{s}\right|\left|\varepsilon_{\tau e}^{d}\right| \cos \left(\phi_{e \tau}^{s}+\phi_{\tau e}^{d}\right)+s_{23}^{2}\left|\varepsilon_{e \mu}^{s}\right|\left|\varepsilon_{\mu e}^{d}\right| \cos \left(\phi_{e \mu}^{s}+\phi_{\mu e}^{d}\right)\right\} \sin ^{2}\left(\Delta_{31}\right), \quad(2.15 \\
= & 2 \sin 2 \theta_{12} \Delta_{21}\left\{-c_{23}\left(\left|\varepsilon_{e \mu}^{s}\right| \sin \phi_{e \mu}^{s}+\left|\varepsilon_{\mu e}^{d}\right| \sin \phi_{\mu e}^{d}\right)\right. \\
& \left.+s_{23}\left(\left|\varepsilon_{e \tau}^{s}\right| \sin \phi_{e \tau}^{s}+\left|\varepsilon_{\tau e}^{d}\right| \sin \phi_{\tau e}^{d}\right)\right\} .
\end{aligned}
$$

The linear coefficients of the terms of order $|\varepsilon|$ in eqs. (2.14), (2.15), (2.16) are the same as given in ref. [46], with a good agreement between the calculated probabilities here and there. However, as it can be seen in the expressions above, here we also include new terms up to second order in $|\varepsilon|$ in the effective neutrino probability. The relevance of these corrections will be discussed later in the paper. In eq. (2.15), note the presence of a term linear in the sine of $\Delta m_{31}^{2} L / 2 E$ which therefore depends on the choice of neutrino mass ordering. This term does not appear in the standard $\bar{\nu}_{e} \rightarrow \bar{\nu}_{e}$ oscillation expression and it can affect the $L / E$ dependence of the probability in the presence of neutrino NSI.

\subsection{Special case of NSI: $\varepsilon_{e \gamma}^{s}=\varepsilon_{\gamma e}^{d *}$}

In this work $^{2}$ we assume that, likewise the mechanisms responsible for production (via $\beta$-decay) and detection (via inverse $\beta$-decay) of reactor antineutrinos are just inverse of each other, this is also true for the associated NSI [46, 47]. This assumption allows us to write $\varepsilon_{\gamma}^{s}=\varepsilon_{\gamma}^{d *} \equiv\left|\varepsilon_{\gamma}\right| \mathrm{e}^{\mathrm{i} \phi_{\gamma}}$ where we drop the universal e index for simplicity. With these assumptions, eq. (2.12) takes the form (keeping the terms up-to the second order in small

\footnotetext{
${ }^{2}$ In the appendix, we have given the effective probability expressions for the physical situations where $\varepsilon_{e \gamma}^{s} \neq \varepsilon_{\gamma e}^{d *}$. In such cases, the spectral analysis of the reactor data becomes inevitable since the NSI parameters not only cause a shift in $\theta_{13}$ i.e. the change of the depth of the first oscillation maximum but also modify the $L / E$ pattern of the oscillation probability due to the shift in its energy. A detailed analysis of the Daya Bay data under such scenarios will be performed in [69].
} 
quantities):

$$
\begin{aligned}
P_{\bar{\nu}_{e}^{s} \rightarrow \bar{\nu}_{e}^{d}} \simeq & \underbrace{1-\sin ^{2} 2 \theta_{13}\left(c_{12}^{2} \sin ^{2} \Delta_{31}+s_{12}^{2} \sin ^{2} \Delta_{32}\right)-c_{13}^{4} \sin ^{2} 2 \theta_{12} \sin ^{2} \Delta_{21}}_{\text {Standard Model terms }} \\
& +\underbrace{4\left|\varepsilon_{e}\right| \cos \phi_{e}+4\left|\varepsilon_{e}\right|^{2}+2\left|\varepsilon_{e}\right|^{2} \cos 2 \phi_{e}+2\left|\varepsilon_{\mu}\right|^{2}+2\left|\varepsilon_{\tau}\right|^{2}}_{\text {non-oscillatory NSI terms }} \\
& -\underbrace{4\left\{s_{23}^{2}\left|\varepsilon_{\mu}\right|^{2}+c_{23}^{2}\left|\varepsilon_{\tau}\right|^{2}+2 s_{23} c_{23}\left|\varepsilon_{\mu}\right|\left|\varepsilon_{\tau}\right| \cos \left(\phi_{\mu}-\phi_{\tau}\right)\right\} \sin ^{2} \Delta_{31}}_{\text {oscillatory NSI terms }} \\
& -\underbrace{4\left\{2 s_{13}\left[s_{23}\left|\varepsilon_{\mu}\right| \cos \left(\delta-\phi_{\mu}\right)+c_{23}\left|\varepsilon_{\tau}\right| \cos \left(\delta-\phi_{\tau}\right)\right]\right\} \sin ^{2} \Delta_{31}}_{\text {oscillatory NSI terms }} .
\end{aligned}
$$

For this special case of NSI parameters, there is no linear sine-dependent term in eq. (2.17) and two striking features are emerging from the effective probability expression which are responsible for a change in the oscillation amplitude. First we can see the presence of some non-oscillatory NSI terms which are independent of $L$ and $E$ and are given by

$$
1+4\left|\varepsilon_{e}\right| \cos \phi_{e}+4\left|\varepsilon_{e}\right|^{2}+2\left|\varepsilon_{e}\right|^{2} \cos 2 \phi_{e}+2\left|\varepsilon_{\mu}\right|^{2}+2\left|\varepsilon_{\tau}\right|^{2},
$$

and, second, there is a shift in the effective 1-3 mixing angle due to oscillatory NSI terms which can be written as

$$
\begin{aligned}
s_{13}^{2} \rightarrow & s_{13}^{2}+s_{23}^{2}\left|\varepsilon_{\mu}\right|^{2}+c_{23}^{2}\left|\varepsilon_{\tau}\right|^{2}+2 s_{23} c_{23}\left|\varepsilon_{\mu}\right|\left|\varepsilon_{\tau}\right| \cos \left(\phi_{\mu}-\phi_{\tau}\right) \\
& +2 s_{13}\left[s_{23}\left|\varepsilon_{\mu}\right| \cos \left(\delta-\phi_{\mu}\right)+c_{23}\left|\varepsilon_{\tau}\right| \cos \left(\delta-\phi_{\tau}\right)\right] .
\end{aligned}
$$

These two features, which are brought about by the NSI parameters, are responsible for a shift in the oscillation amplitude without distorting the $L / E$ pattern of the oscillation probability as can be clearly seen from figure 1 and figure 2 that we will discuss in the next section. Eq. (2.19) suggests that it will be quite challenging to discriminate the effect of true $\theta_{13}$ and NSI parameters in the modern reactor experiments. It is also interesting to note that there are some CP conserving terms in eq. (2.19) which come into the picture due to the presence of NSI parameters. One of the most important consequences of the new non-oscillatory NSI terms (see eq. (2.18)) is that they can cause a flavor transition at the source $(L=0)$ even before neutrinos start to oscillate. In the literature, this feature is known as "zero-distance" effect [46, 70]. In modern reactor experiments, this effect can be probed using the near detectors which are placed quite close to the source.

For definiteness, in this work we have restricted our analysis to the following choices of the NSI parameters:

- Lepton number conserving non-universal NSI parameters which depend on the flavor characterizing the violation of weak universality. Under this category, we study the following two cases:

1. Considering only the NSI parameters $\left|\varepsilon_{e}\right|$ and $\phi_{e}$ which are associated with $\bar{\nu}_{e}$. In the presence of these flavor conserving NSI parameters, eq. (2.17) takes the form:

$$
P_{\bar{\nu}_{e}^{s} \rightarrow \bar{\nu}_{e}^{d}}^{\mathrm{NSI}} \simeq P_{\bar{\nu}_{e} \rightarrow \bar{\nu}_{e}}^{\mathrm{SM}}+4\left|\varepsilon_{e}\right| \cos \phi_{e}+4\left|\varepsilon_{e}\right|^{2}+2\left|\varepsilon_{e}\right|^{2} \cos 2 \phi_{e} .
$$


At first order in $\left|\varepsilon_{e}\right|$ and neglecting the effect of the solar mass splitting, the new non-oscillatory NSI terms appearing at the survival probability produce a total shift in the effective $\theta_{13}$ mixing angle given by:

$$
\tilde{s}_{13}^{2} \approx s_{13}^{2}-\frac{\left|\varepsilon_{e}\right| \cos \phi_{e}}{\sin ^{2} \Delta_{31}} .
$$

This expression will be very useful to discuss the behavior of the effective probability as well as the correlations between $\theta_{13}$ and the NSI parameters in the next subsections.

2. Considering only the NSI parameters $\left|\varepsilon_{\mu}\right|$ and $\phi_{\mu}$ which are associated with $\bar{\nu}_{\mu}$. In the presence of these flavor violating NSI parameters, eq. (2.17) takes the form:

$$
P_{\bar{\nu}_{e}^{s} \rightarrow \bar{\nu}_{e}^{d}}^{\mathrm{NSI}} \simeq P_{\bar{\nu}_{e} \rightarrow \bar{\nu}_{e}}^{\mathrm{SM}}+2\left|\varepsilon_{\mu}\right|^{2}-4\left\{s_{23}^{2}\left|\varepsilon_{\mu}\right|^{2}+2 s_{13} s_{23}\left|\varepsilon_{\mu}\right| \cos \left(\delta-\phi_{\mu}\right)\right\} \sin ^{2} \Delta_{31} .
$$

Note that, for the NSI parameters $\left|\varepsilon_{\tau}\right|$ and $\phi_{\tau}$ which are associated with $\bar{\nu}_{\tau}$, the effective survival probability will be exactly the same as eq. (2.22) with the replacements $\left|\varepsilon_{\mu}\right| \rightarrow\left|\varepsilon_{\tau}\right|$ and $\phi_{\mu} \rightarrow \phi_{\tau}$ provided that the 2-3 mixing angle is maximal ,i.e., $\sin ^{2} \theta_{23}=0.5$. As before, the new non-standard oscillatory terms in the neutrino transition probability may be interpreted as a global redefinition of the effective $\theta_{13}$ mixing angle:

$$
\tilde{s}_{13}^{2} \approx s_{13}^{2}+2 s_{13} s_{23}\left|\varepsilon_{\mu}\right| \cos \left(\delta-\phi_{\mu}\right) .
$$

- Lepton number conserving universal NSI parameters which do not depend on flavor. In this case, we have $\left|\varepsilon_{e}\right|=\left|\varepsilon_{\mu}\right|=\left|\varepsilon_{\tau}\right|=|\varepsilon|$ and $\phi_{e}=\phi_{\mu}=\phi_{\tau}=\phi$ and the probability in eq. (2.17) takes the form:

$$
\begin{aligned}
P_{\bar{\nu}_{e}^{s} \rightarrow \bar{\nu}_{e}^{d}}^{\mathrm{NSI}} \simeq & P_{\bar{\nu}_{e} \rightarrow \bar{\nu}_{e}}^{\mathrm{SM}}+4|\varepsilon| \cos \phi+2|\varepsilon|^{2}(4+\cos 2 \phi) \\
& -4\left\{|\varepsilon|^{2}+2 s_{23} c_{23}|\varepsilon|^{2}+2 s_{13}|\varepsilon| \cos (\delta-\phi)\left(s_{23}+c_{23}\right)\right\} \sin ^{2} \Delta_{31} .
\end{aligned}
$$

In this case, the effective mixing angle in the presence of oscillatory and nonoscillatory NSI terms will be given by:

$$
\tilde{s}_{13}^{2} \approx s_{13}^{2}-|\varepsilon|\left[\frac{\cos \phi}{\sin ^{2} \Delta_{31}}-2 s_{13}\left(s_{23}+c_{23}\right) \cos (\delta-\phi)\right] .
$$

As stated above, the expressions given in this subsection to illustrate the shift in the effective reactor angle in the presence of NSI, eqs. (2.21), (2.23) and (2.25), contain only first order corrections in the NSI couplings. Note, however, that terms of second order in $|\varepsilon|$ have been included in all the numerical results shown along the paper. Here we will briefly discuss the relevance of second order corrections in our analysis. Clearly, these corrections to the effective neutrino probability are only significant in the cases where first order corrections are very small or totally cancelled. This happens for $\phi_{e}= \pm 90^{\circ}$ in the case of electron-NSI couplings, for $\left(\delta-\phi_{\mu, \tau}\right)= \pm 90^{\circ}$ in the case of muon/tau-NSI couplings 


\begin{tabular}{|l|l|l|l|l|l|l|}
\hline Parameter & $\sin ^{2} \theta_{12}$ & $\sin ^{2} \theta_{23}$ & $\sin ^{2} \theta_{13}$ & $\Delta m_{21}^{2}\left(\mathrm{eV}^{2}\right)$ & $\Delta m_{31}^{2}\left(\mathrm{eV}^{2}\right)$ & $\delta$ \\
\hline Value & 0.32 & 0.5 & 0.023 & $7.6 \times 10^{-5}$ & $2.55 \times 10^{-3}$ & $0-2 \pi$ \\
\hline
\end{tabular}

Table 1. Benchmark values of the neutrino oscillation parameters used in this work, taken from refs. $[5,71]$.
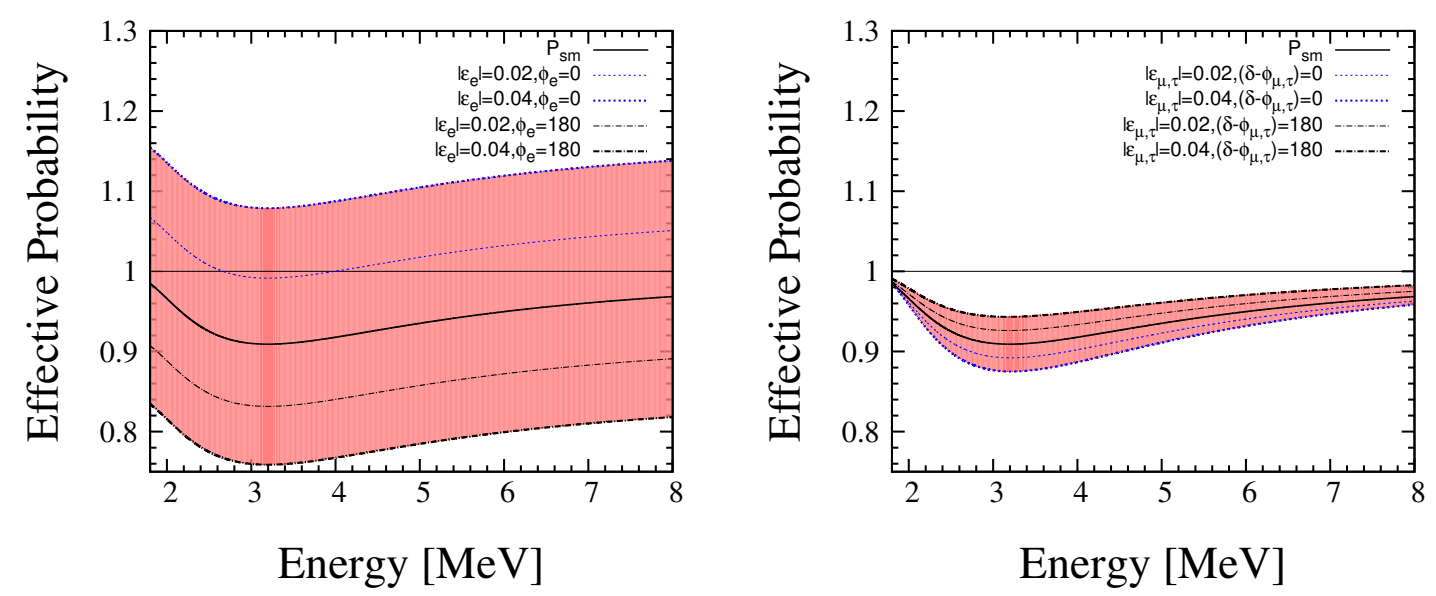

Figure 1. Effective $\bar{\nu}_{e}^{s} \rightarrow \bar{\nu}_{e}^{d}$ survival probability as a function of neutrino energy in the presence of NSI with $L=1.58 \mathrm{~km}$. The band in the left panel has been generated by varying $\left|\varepsilon_{e}\right|$ in the range $[0,0.04]$ and $\phi_{e}$ over the range $\left[-180^{\circ}, 180^{\circ}\right]$ simultaneously. The simultaneous variation of $\left|\varepsilon_{\mu, \tau}\right|$ in the range $[0,0.04]$ and $\left(\delta-\phi_{\mu, \tau}\right)$ over the range $\left[-180^{\circ}, 180^{\circ}\right]$ is responsible for the band in the right panel. In both the panels, the solid black lines depict the probability without new physics involved (SM case).

and for $\left(\delta=0, \phi= \pm 90^{\circ}\right)$ in the flavour-universal case. From eqs. (2.20), (2.22) and (2.24), it is straightforward to evaluate the size of the second order terms for the three cases under study. Taking into account the baselines and neutrino energies probed at the Daya Bay experiment, we find that second order corrections (for vahishing first order corrections) are approximately given by: $0.5\left|\varepsilon_{e}\right|^{2}$ (electron-NSI case), $0.05\left|\varepsilon_{\mu, \tau}\right|^{2}$ (muon/tau-NSI case) and $0.4|\varepsilon|^{2}$ (flavour-universal-NSI case). The small size of the corrections in the muon/tau-NSI, one order of magnitude smaller than for the two other cases, comes from the smallness of the coefficient responsible for second order corrections in the expression of the effective reactor angle, $\left(\frac{1}{2 \sin ^{2} \Delta_{31}}-s_{23}^{2}\right)$, very close to zero for the energies and baselines studied in Daya Bay. This result can be observed in the correlation plots in figures 3 and 4 (see dashed blue line) in section 2.4. There, one sees that second order corrections are small but visible for electron-NSI and flavour-universal case, while they are almost negligible for the muon/tau-NSI case. The impact of the second order corrections over the results presented in this work is discussed in section 7 . 


\subsection{Impact of the NSI parameters on the effective probability}

Now we will study the possible impact of the NSI parameters at the effective probability level. Table 1 depicts the benchmark values of the various oscillation parameters that are considered to generate the oscillation probability plots. These choices of the oscillation parameters are in close agreement with the best-fit values that have been obtained in the recent global fits of the world neutrino oscillation data [5-7]. Here we would like to mention that for the 2-3 mixing angle, we have taken the maximal value i.e. $\sin ^{2} \theta_{23}=0.5$, though in the global fit studies, there is a slight hint for a non-maximal value of $\theta_{23}$. We have also assumed normal mass ordering, i.e., $\Delta m_{31}^{2}$ positive. In figure 1, we present the standard and the NSI-modified three-flavor oscillation effective probability as a function of the electron antineutrino energy with a source-detector distance of $1.58 \mathrm{~km}$. In the left panel of figure 1 , the band shows how the probability changes if we vary $\left|\varepsilon_{e}\right|$ in the range $[0,0.04]$ and $\phi_{e}$ over the range $\left[-180^{\circ}, 180^{\circ}\right]$ simultaneously. For the NSI parameters which are only associated with $\bar{\nu}_{e}$, the probability is independent of the CP phase $\delta$ (see eq. (2.13) and eq. (2.20)). The solid black line shows the standard oscillation probability without the NSI terms (see eq. (2.13)). The other four lines have been drawn considering particular choices of $\left|\varepsilon_{e}\right|$ and $\phi_{e}$ which are mentioned in the figure legends. If $\phi_{e}=180^{\circ}$, then the oscillation probability is less compared to the standard value because the contribution from the nonoscillatory NSI terms takes the form $-4\left|\varepsilon_{e}\right|+6\left|\varepsilon_{e}\right|^{2}$ which always gives an overall negative contribution to the full probability if $\left|\varepsilon_{e}\right| \leq 0.66$. On the other hand, if we consider $\phi_{e}=0^{\circ}$, then the oscillation probability is above the standard value because the contribution from the non-oscillatory terms takes the form $4\left|\varepsilon_{e}\right|+6\left|\varepsilon_{e}\right|^{2}$ which always gives an overall positive contribution to the full probability for any choice of $\left|\varepsilon_{e}\right|$. We can also see that even for a small value of $\left|\varepsilon_{e}\right|$ of 0.02 , the effective oscillation probability can be more than unity for most of the energies of interest. This is the sign of the non-unitarity effects [68, 72-75], caused by the presence of neutrino NSI at the source and detector of reactor experiments. In the right panel of figure 1 , the band shows the changes in the effective probability after varying $\left|\varepsilon_{\mu, \tau}\right|$ in the range $[0,0.04]$ and $\left(\delta-\phi_{\mu, \tau}\right)$ over the range $\left[-180^{\circ}, 180^{\circ}\right]$ simultaneously. Note that in eq. (2.22), the phases appear in the form of cosine of $\left(\delta-\phi_{\mu, \tau}\right)$ and also the NSI terms have a non-trivial $L / E$ dependency. The standard oscillation probability without the NSI terms is shown by the solid black line and the other four lines have been drawn considering particular choices of $\left|\varepsilon_{\mu, \tau}\right|$ and $\left(\delta-\phi_{\mu, \tau}\right)$ which are mentioned in the figure legends. If $\left(\delta-\phi_{\mu, \tau}\right)=0^{\circ}\left(180^{\circ}\right)$, then the effective oscillation probability is less (more) compared to the standard value for almost all the choices of neutrino energy as opposed to the case of the NSI parameters associated with $\bar{\nu}_{e}$.

Figure 2 shows the impact of the NSI parameters at the probability level with $L=1.58$ $\mathrm{km}$ for the flavor-universal NSI case where we consider $\left|\varepsilon_{e}\right|=\left|\varepsilon_{\mu}\right|=\left|\varepsilon_{\tau}\right|=|\varepsilon|$ and $\phi_{e}=$ $\phi_{\mu}=\phi_{\tau}=\phi$. In this plot, the dark salmon region has been generated by varying the NSI parameter $|\varepsilon|$ in the range $[0,0.05]$ and $\phi$ in the range $\left[-180^{\circ}, 180^{\circ}\right]$ simultaneously, keeping the CP phase $\delta$ fixed to $0^{\circ}$. Next, we vary $\delta$ in its entire range from $-180^{\circ}$ to $180^{\circ}$ along with the NSI parameters $|\varepsilon|$ and $\phi$ and obtain the extended probability band in the form of the light grey region. In figure 2 , the solid black line depicts the standard probability without 


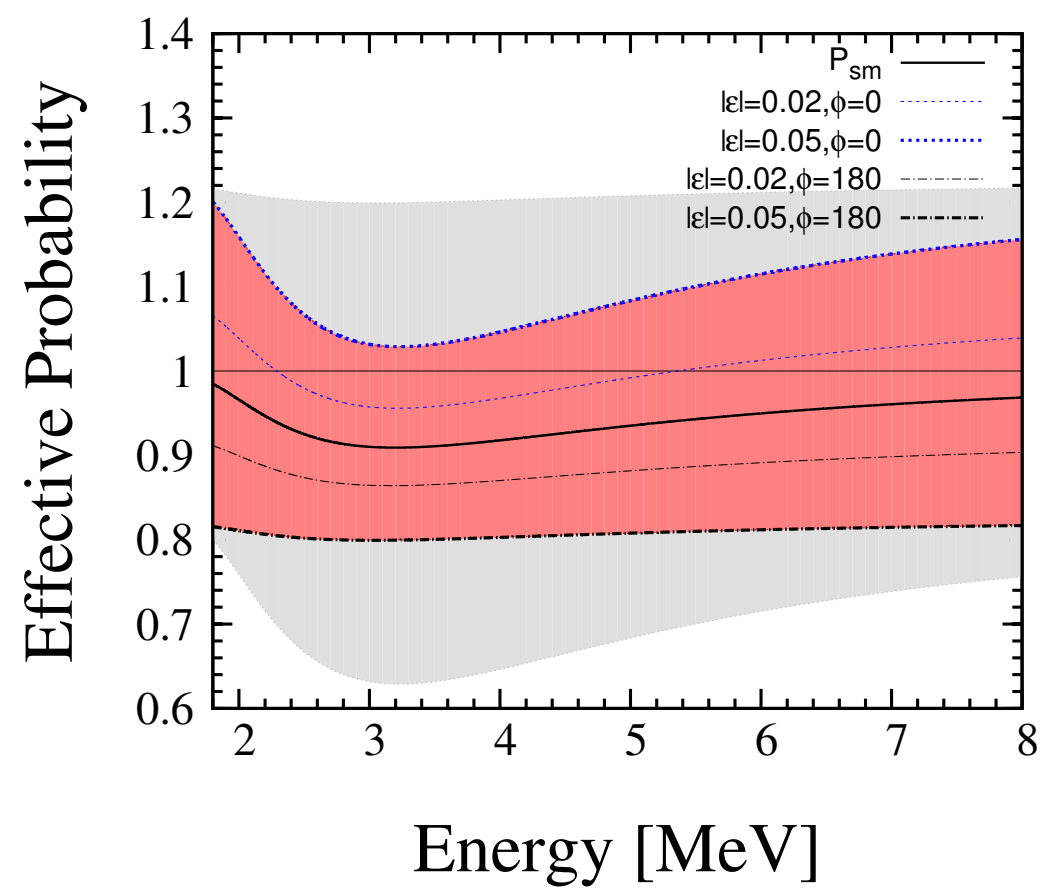

Figure 2. Effective $\bar{\nu}_{e}^{s} \rightarrow \bar{\nu}_{e}^{d}$ survival probability as a function of neutrino energy with $L=1.58$ $\mathrm{km}$ for the flavor-universal NSI case (see section 2.2 for details). The dark salmon region shows the combined effect of the variation of the new physics parameters $|\varepsilon|$ and $\phi$ with $\delta=0^{\circ}$. The extended probability band in light grey has been obtained by varying the CP phase $\delta$ in the range $\left[-180^{\circ}\right.$, $\left.180^{\circ}\right]$ along with the other two parameters $|\varepsilon|$ and $\phi$. The solid black line displays the probability without new physics involved (SM case).

considering the NSI parameters. The other four lines in this plot display the effective oscillation probability for particular combinations of $|\varepsilon|$ and $\phi$ with $\delta=0^{\circ}$ which are mentioned in the figure legends. It is quite clear from eq. (2.24) that the non-oscillatory terms dominate over the oscillatory terms in the flavor-universal NSI case. Therefore, the dark salmon region of figure 2 closely resembles the left panel of figure 1 where we consider the NSI parameters which are only associated with $\bar{\nu}_{e}$, namely $\left|\varepsilon_{e}\right|$ and $\phi_{e}$. Next, we discuss the possible correlations between the NSI parameters and $\theta_{13}$ with the help of iso-probability plots.

\subsection{Correlations between NSI parameters and $\theta_{13}$ : iso-probability plots}

We consider the neutrino energy $E=4 \mathrm{MeV}$ and the source-detector distance $L=1.58 \mathrm{~km}$ to draw the iso-probability surface plots. Left panel of figure 3 shows the iso-probability surface contours in the $\left(\sin ^{2} \theta_{13}-\left|\varepsilon_{e}\right|\right)$ plane for four different choices of $\phi_{e}$ considering $\sin ^{2} \theta_{13}$ $=0.023$ and $\left|\varepsilon_{e}\right|=0$ as best-fit choices. Our best-fit choices correspond to the standard oscillation probability without considering the NSI parameters as given by eq. (2.13). Now as we consider the finite value of $\left|\varepsilon_{e}\right|$ with $\phi_{e}=0^{\circ}$ (see the solid red line), the NSI terms increase the overall probability (see eq. (2.20)). Then, we need to increase the value of 

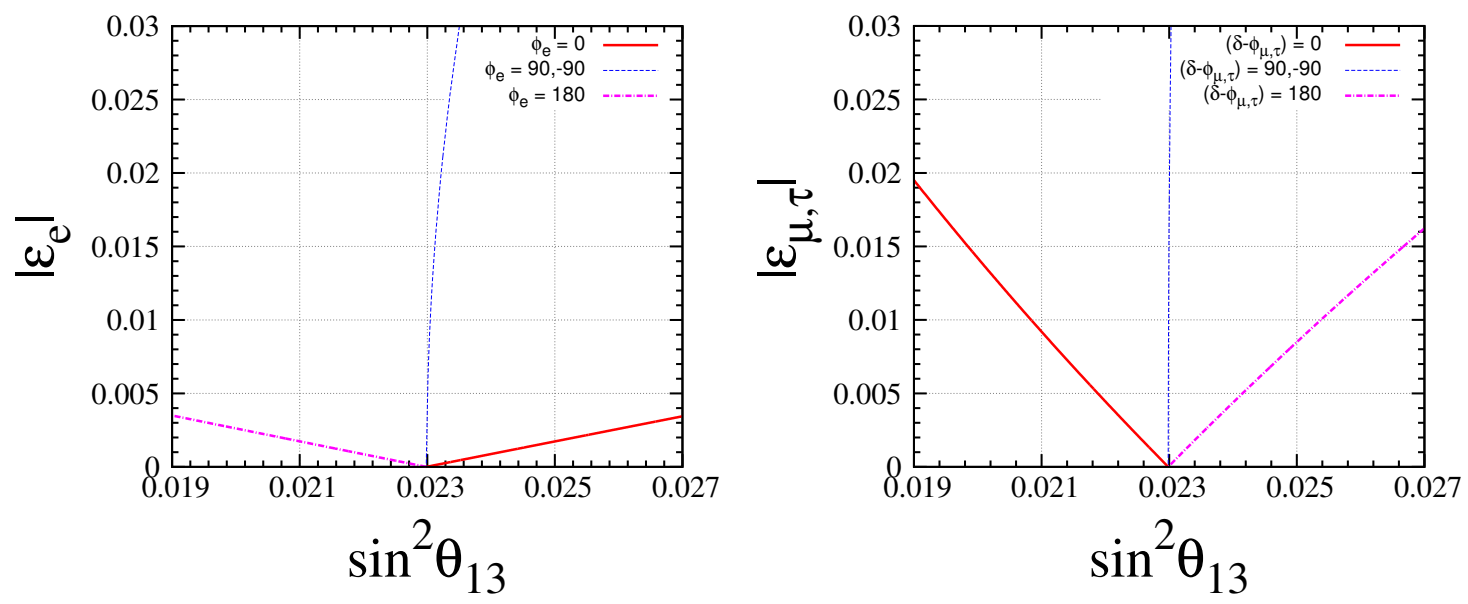

Figure 3. Left panel shows the iso-probability surface contours in the $\left(\sin ^{2} \theta_{13}-\left|\varepsilon_{e}\right|\right)$ plane for different choices of $\phi_{e}$ as mentioned in the figure legends. Here we consider $\sin ^{2} \theta_{13}=0.023$ and $\left|\varepsilon_{e}\right|$ $=0$ as benchmark choices. Right panel displays the same in the $\left(\sin ^{2} \theta_{13}-\left|\varepsilon_{\mu, \tau}\right|\right)$ plane for different choices of $\left(\delta-\phi_{\mu, \tau}\right)$ considering $\sin ^{2} \theta_{13}=0.023$ and $\left|\varepsilon_{\mu, \tau}\right|=0$ as true choices. For both the panels, we consider a fixed neutrino energy $E=4 \mathrm{MeV}$ and the baseline $L=1.58 \mathrm{~km}$.

$\sin ^{2} \theta_{13}$ to reduce the SM probability in order to compensate the enhancement due to the NSI contribution. For $\phi_{e}=180^{\circ}$ case (see the dot-dashed magenta line), a finite value of $\left|\varepsilon_{e}\right|$ decreases the overall probability demanding a lower value of $\sin ^{2} \theta_{13}$ so as to enhance the contribution from the standard probability. In the cases with $\phi_{e}=90^{\circ}$ or $-90^{\circ}$ (see the dashed blue line), the non-oscillatory NSI term which is linear in $\left|\varepsilon_{e}\right|$ drops out from the probability expression and the remaining NSI contribution is $2\left|\varepsilon_{e}\right|^{2}$ (see eq. (2.20)). Due to this weak quadratic dependence on $\left|\varepsilon_{e}\right|$, a very large value of $\left|\varepsilon_{e}\right|$ is needed to compensate even a very small increment in $\sin ^{2} \theta_{13}$. Right panel displays the same in the $\left(\sin ^{2} \theta_{13^{-}}\left|\varepsilon_{\mu, \tau}\right|\right)$ plane for different choices of $\left(\delta-\phi_{\mu, \tau}\right)$. Here $\sin ^{2} \theta_{13}=0.023$ and $\left|\varepsilon_{\mu, \tau}\right|=0$ are considered as true choices. In this panel, the combination of phases $\left(\delta-\phi_{\mu, \tau}\right)$ shows opposite features for $0^{\circ}$ and $180^{\circ}$ as compared to $\phi_{e}$ in the left panel. Note that the impact of $\left|\varepsilon_{\mu, \tau}\right|$ on $\sin ^{2} \theta_{13}$ is weaker compared to $\left|\varepsilon_{e}\right|$. If we examine the terms which are linear in $\left|\varepsilon_{e}\right|$ (see eq. (2.20)) and $\left|\varepsilon_{\mu, \tau}\right|$ (see eq. (2.22)) then we can see that the contribution coming from $\left|\varepsilon_{\mu, \tau}\right|$ is $\sin \theta_{13}$ suppressed even if we work at the first oscillation maximum. For $\left(\delta-\phi_{\mu, \tau}\right)$ $=90^{\circ}$ or $-90^{\circ}$, the $\left|\varepsilon_{\mu, \tau}\right|$-dependent terms completely disappear from eq. (2.22) if $\sin ^{2} \theta_{23}=$ 0.5 and $\sin ^{2} \Delta_{31}=1$. Therefore, we do not see any correlation between $\sin ^{2} \theta_{13}$ and $\left|\varepsilon_{\mu, \tau}\right|$ for $\left(\delta-\phi_{\mu, \tau}\right)=90^{\circ}$ or $-90^{\circ}$ in the right panel of figure 3 .

In figure 4 , we show the correlation between $\sin ^{2} \theta_{13}$ and $|\varepsilon|$ for the flavor-universal NSI case with the help of iso-probability surface contours. In the left panel, we consider four different choices of the $\mathrm{CP}$ phase $\delta$ keeping $\phi$ fixed to $0^{\circ}$. In the right panel, we take five different choices of $\phi$ assuming $\delta=0^{\circ}$. In both panels, the iso-probability surface contours are the same for $90^{\circ}$ and $-90^{\circ}$ choices of phases (see the dashed blue lines) because the phases appear in the form of cosines in eq. (2.24). For the $\phi=0^{\circ}$ case (left panel), the contribution 

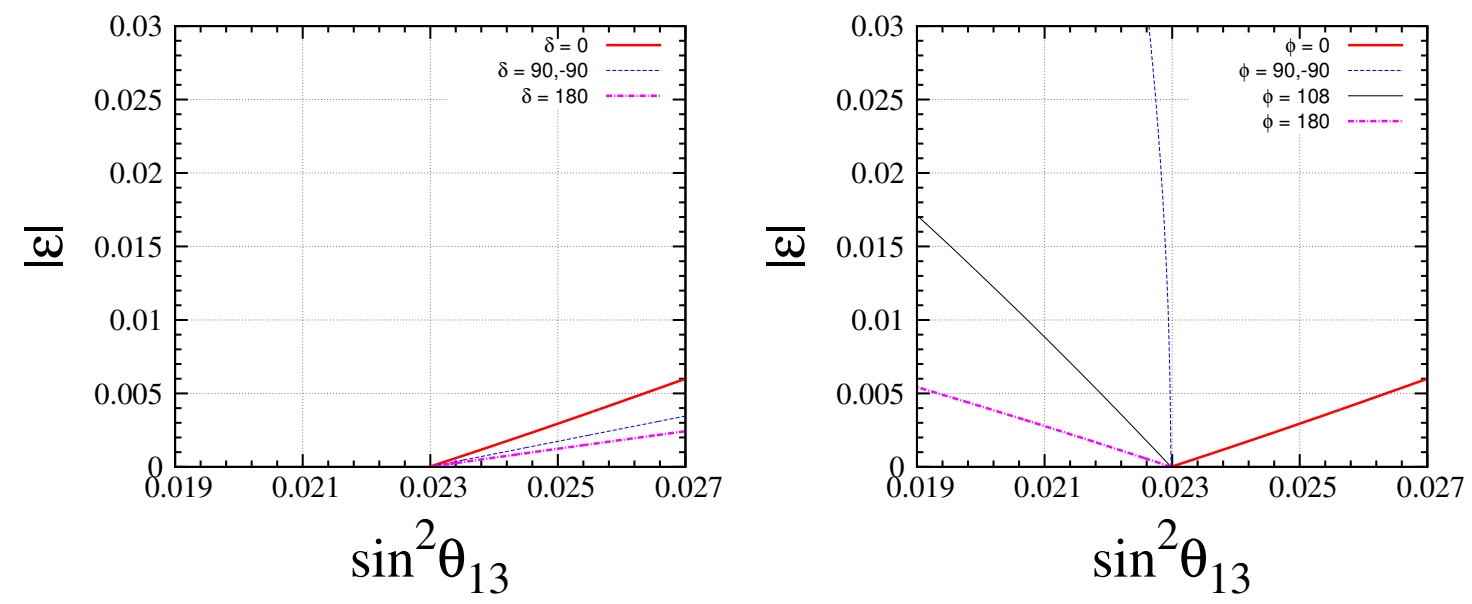

Figure 4. Left panel shows the iso-probability surface contours for the flavor-universal NSI case in the $\left(\sin ^{2} \theta_{13}-|\varepsilon|\right)$ plane for different choices of the $\mathrm{CP}$ phase $\delta$ with $\phi=0^{\circ}$. Here we consider $\sin ^{2} \theta_{13}=0.023$ and $|\varepsilon|=0$ as benchmark choices. Right panel displays the same for various choices of $\phi$ assuming $\delta=0^{\circ}$. For both the panels, we take a fixed neutrino energy $E=4 \mathrm{MeV}$ and the baseline $L=1.58 \mathrm{~km}$.

from the non-oscillatory NSI terms is maximum and takes the form $4|\varepsilon|+10|\varepsilon|^{2}$. Thus, it enhances the overall oscillation probability to a great extent even for a small value of $|\varepsilon|$. Now to compensate this enhancement, we need to increase the value of $\sin ^{2} \theta_{13}$ to reduce the contribution coming from the standard survival oscillation probability. Right panel of figure $4\left(\delta=0^{\circ}\right.$ case) closely resembles the left panel of figure 3 because for the flavor-universal NSI case, the contribution from the non-oscillatory NSI terms dominates which is also true for the NSI parameters associated with $\bar{\nu}_{e}$ and also these NSI parameters are $\delta$ independent. In the right panel, we present a special case of $\phi=108^{\circ}$ to explain the features emerging from the extreme right panel of figure 7 (see later in section 4) where we have displayed the allowed region in $\left(\sin ^{2} \theta_{13}-|\varepsilon|\right)$ plane using the current data from the Daya Bay experiment allowing the flavor-universal NSI phase $\phi$ to vary in the entire range of $\left[-180^{\circ}, 180^{\circ}\right]$ with the CP phase $\delta$ to be fixed to $0^{\circ}$. Eq. (2.24) suggests that we can always choose some values of $\phi$ such that the non-oscillatory terms are canceled. This happens, for instance, for $\phi=108^{\circ}$ and $|\varepsilon|=0.206$. In this case, the dominant oscillatory NSI terms give a negative contribution to the neutrino survival probability that, to be compensated, requires an enhancement of the standard survival probability by reducing the value of $\sin ^{2} \theta_{13}$ by the same quantity. This is exactly the feature that we can see for $\phi=108^{\circ}$ case.

\section{Data analysis of modern reactor experiments}

Reactor antineutrinos are produced by the fission of the isotopes ${ }^{235} \mathrm{U},{ }^{239} \mathrm{Pu},{ }^{241} \mathrm{Pu}$ and ${ }^{238} \mathrm{U}$ contributing to the neutrino flux with a certain fission fraction $f_{k}$. Reactor antineutrinos are detected via inverse $\beta$-decay process (IBD), $\overline{\nu_{e}}+p \rightarrow e^{+}+n$. The technique used is a delayed coincidence between two gamma rays: one coming from the positron (prompt 
signal) and the other coming from the neutron capture in the innermost part of the antineutrino detector $(\mathrm{AD})$, containing gadolinium-doped liquid scintillator. The light created is collected by the photo-multipliers (PMTs) located in the outermost mineral oil-region. The antineutrino energy $E_{\bar{\nu}}$ is reconstructed from the positron prompt energy $E_{\text {prompt }}$ following the relation: $E_{\bar{\nu}}=E_{\text {prompt }}+\bar{E}_{n}+0.78 \mathrm{MeV}$, where $\bar{E}_{n}$ is the average neutron recoil energy. The expected number of IBD events at the $d$-th detector, $T_{d}$, can be estimated summing up the contributions of all reactors to the detector:

$$
\begin{aligned}
T_{d} & =\sum_{r} T_{r d}= \\
& =\sum_{r} \epsilon_{d} \frac{N_{p}}{4 \pi L_{r d}^{2}} \frac{P_{t h}^{r}}{\sum_{k} f_{k}\left\langle E_{k}\right\rangle} \sum_{k} f_{k} \int_{0}^{\infty} d E \Phi_{k}(E) \sigma_{I B D}(E) P_{e e}\left(E, L_{r d}\right),
\end{aligned}
$$

where $N_{p}$ is the number of protons in the target volume, $P_{t h}^{r}$ is the reactor thermal power, $\epsilon_{d}$ denotes the efficiency of the detector and $\left\langle E_{k}\right\rangle$ is the energy release per fission for a given isotope $k$ taken from ref. [76]. The neutrino survival probability $P_{e e}$ depends also on the distance from $r$-th reactor to $d$-th detector, $L_{r d}$. For the antineutrino flux prediction $\Phi_{k}(E)$ we use the parameterization given in ref. [77] as well as the new normalization for reactor antineutrino fluxes updated in ref. [78]. The inverse beta decay cross section $\sigma_{\mathrm{IBD}}\left(E_{\nu}\right)$ is taken from ref. [79].

\section{Daya Bay experiment}

Daya Bay is a reactor neutrino experiment with several antineutrino detectors (ADs), arranged in three experimental halls (EHs). Electron antineutrinos are generated in six reactor cores, distributed in pairs, with equal thermal power $\left(\mathrm{P}_{t h}^{r}=2.9 \mathrm{GW}_{t h}\right)$ and detected in the EHs. The effective baselines are $512 \mathrm{~m}$ and $561 \mathrm{~m}$ for the near halls EH1 and EH2 and $1579 \mathrm{~m}$ for the far hall EH3 [2]. With this near-far technology Daya Bay has minimized the systematic errors coming from the ADs and thus provided until now the most precise determination of the reactor mixing angle. In the last Neutrino conference, Daya Bay has reported its preliminary results considering 621 days of data taking combining their results for two different experimental setups [3]: one with six ADs as it was published in ref. [2] and the other after the installation of two more detectors, eight ADs in total. This new combined data set has four times more statistics in comparison with the previous Daya Bay results. Thus, the precision in the determination of the reactor mixing angle has been improved, and it is now of the order of $6 \%$.

In this work we will consider the most recent data release by the Daya Bay Collaboration described above and we will concentrate on the total observed rates at each detector, that will be analyzed using the following $\chi^{2}$ expression:

$$
\begin{aligned}
\chi^{2}= & \sum_{d=1}^{8} \frac{\left[M_{d}-T_{d}\left(1+a_{\mathrm{norm}}+\sum_{r} \omega_{r}^{d} \alpha_{r}+\xi_{d}\right)+\beta_{d}\right]^{2}}{M_{d}+B_{d}} \\
& +\sum_{r=1}^{6} \frac{\alpha_{r}^{2}}{\sigma_{r}^{2}}+\sum_{d=1}^{8}\left(\frac{\xi_{d}^{2}}{\sigma_{d}^{2}}+\frac{\beta_{d}^{2}}{\sigma_{B}^{2}}\right)+\frac{a_{\mathrm{norm}}^{2}}{\sigma_{a}^{2}} .
\end{aligned}
$$


Here $T_{d}$ corresponds to the theoretical prediction in eq. (3.1), $M_{d}$ is the measured number of events at the $d$-th $\mathrm{AD}$ with its backgrounds $\left(B_{d}\right)$ subtracted and $\omega_{r}^{d}$ is the fractional contribution of the $r$-th reactor to the $d$-th $\mathrm{AD}$ number of events, determined by the baselines $L_{r d}$ and the total thermal power of each reactor. The pull parameters, used to include the systematical errors in the analysis, are given by the set $\left(\alpha_{r}, \xi_{d}, \beta_{d}\right)$ representing the reactor, detector and background uncertainties with the corresponding set of errors $\left(\sigma_{r}, \sigma_{d}, \sigma_{B}\right)$. Uncertainties in the reactor related quantities are included in $\sigma_{r}(0.8 \%)$ while the uncorrelated combined uncertainties in the ADs are included in $\sigma_{d}(0.2 \%) . \sigma_{B}$ is the quadratic sum of the background uncertainties taken from ref. [3]. Finally, we also consider an absolute normalization factor $a_{\text {norm }}$ to account for the uncertainty in the total normalization of events at the ADs, given by $\sigma_{a}$, and coming for instance from uncertainties in the normalization of reactor antineutrino fluxes. In our analysis we will follow two different approaches concerning this parameter. In section 4 we will take it equal to zero, assuming perfect knowledge of the events normalization. This hypothesis will be relaxed in section 5, where we will allow for a non-zero normalization factor in the statistical analysis, being determined from the fit to the Daya Bay data. As we will see, the results obtained in our analysis are strongly correlated with the treatment of the total normalization of reactor neutrino events in the statistical analysis of Daya Bay data and therefore it is of crucial importance to do a proper treatment of this factor.

\section{Bounds on NSI from Daya Bay without normalization error}

In this section we will present the bounds on the NSI couplings we have obtained using current Daya Bay reactor data. In all the results, we have assumed maximal 2-3 mixing and we have marginalized over atmospheric splitting with a prior of $3 \%$. For definiteness we will start considering only the couplings relative to electron neutrino: $\left(\left|\varepsilon_{e}\right|, \phi_{e}\right)$, for what we will switch all the other NSI parameters to zero. Next we will do the same for $\left(\left|\varepsilon_{\mu}\right|, \phi_{\mu}\right)$ and $\left(\left|\varepsilon_{\tau}\right|, \phi_{\tau}\right)$, that are equivalent for maximal value of $\theta_{23}$. Finally, we will consider the possibility of having all NSI couplings with the same value: $\varepsilon_{e}=\varepsilon_{\mu}=\varepsilon_{\tau}=\varepsilon$. In all cases we will discuss the bounds arising from Daya Bay data in comparison with existing bounds. We will also consider the robustness of the $\theta_{13}$ measurement by Daya Bay in the presence of NSI.

\subsection{Constraints on electron-NSI couplings}

According to the expression in eq. (2.20), the effective survival probability in the case when only NSI with electron antineutrinos are considered is independent of the standard CP phase $\delta$. Therefore, in our analysis we consider only two cases, one with the only relevant phase $\phi_{e}$ set to zero, and a second case where we allow this phase to vary freely. Our results are presented in figure 5. From the left panel in this figure, we can confirm the behaviors shown by the iso-probability curves in the section 2.4, namely, the presence of a non-zero $\varepsilon_{e}$ coupling has to be compensated with a slightly larger value of the reactor mixing angle $\theta_{13}$. In this panel one also sees how current Daya Bay data constrain very strongly the magnitude of the NSI coupling $\left|\varepsilon_{e}\right|$, improving the current bound in eq. (1.3) 

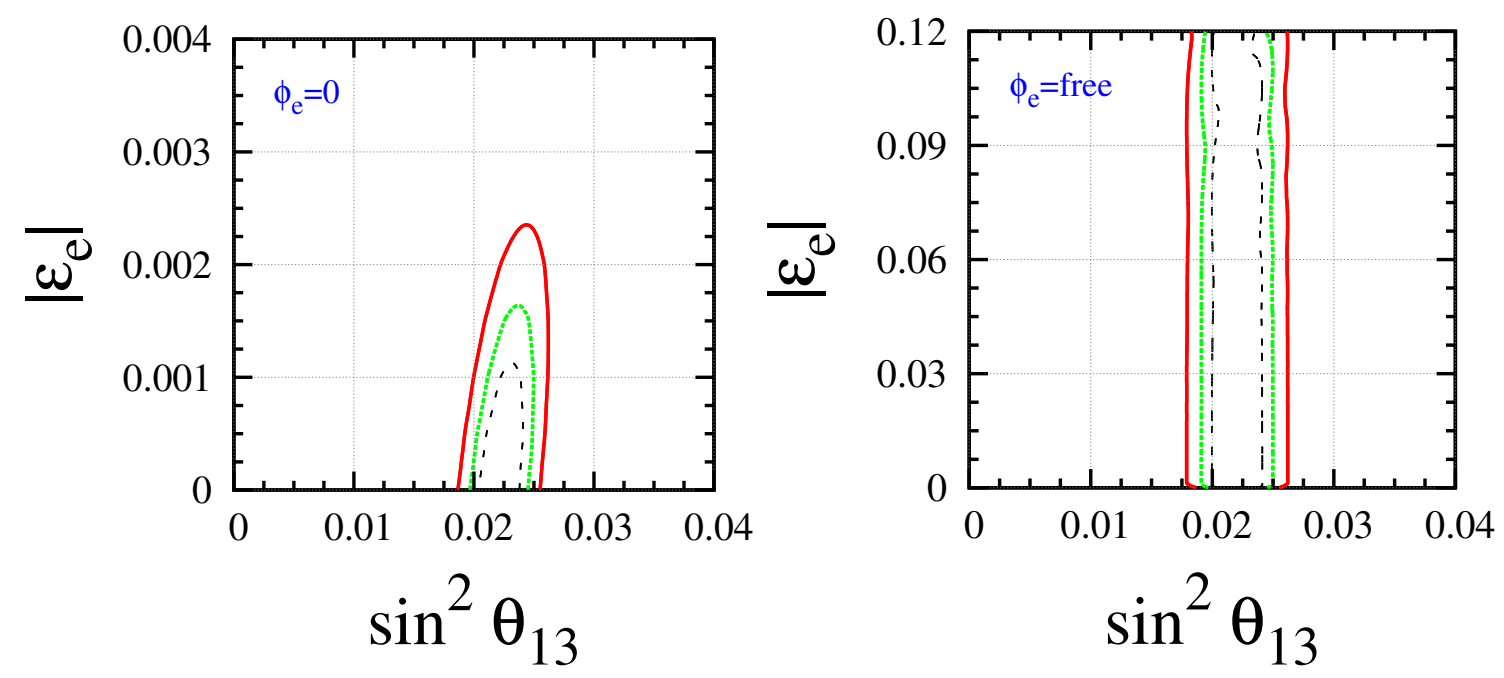

Figure 5. Allowed region in the $\sin ^{2} \theta_{13}-\left|\varepsilon_{e}\right|$ plane. Left panel is obtained by setting the phase $\phi_{e}$ to zero, while in the right panel $\phi_{e}$ is marginalized, varying freely between $-180^{\circ}$ and $180^{\circ}$. The regions correspond to $68 \%$ (black dashed line), $90 \%$ (green line) and 99\% C.L. (red line) for 2 d.o.f.

by one order of magnitude:

$$
\left|\varepsilon_{e}\right| \leq 1.2 \times 10^{-3} \quad \text { (90\% C.L.) } .
$$

However, the situation changes dramatically when the phase $\phi_{e}$ is allowed to vary freely, as shown in the right panel of figure 5. In this case, the strong bound on $\left|\varepsilon_{e}\right|$ disappears due to the presence of a correlation bewteen $\left|\varepsilon_{e}\right|$ and $\cos \phi_{e}$ in the $\left|\varepsilon_{e}\right|$-linear term in the neutrino survival probability (see eq. (2.20)). The presence of second order terms in $\left|\varepsilon_{e}\right|$ is not enough to break this degeneracy and, therefore, the sensitivity to $\left|\varepsilon_{e}\right|$ disappears and no bound can be obtained from reactor data.

Concerning the determination of the reactor mixing angle, the presence of the NSIcoupling with electron antineutrinos results in the following allowed range for $\theta_{13}$ :

$$
0.020 \leq \sin ^{2} \theta_{13} \leq 0.024 \quad(90 \% \text { C.L. }) .
$$

The same interval is obtained for the two panels at figure 5 and it also coincides exactly with the allowed range in absence of NSI. In consequence, we can say that the reactor angle determination by Daya Bay is robust in this specific case.

\subsection{Constraints on muon/tau-NSI couplings}

In this subsection we present the results obtained considering only the NSI parameters associated with muon and tau neutrinos. As we have discussed in section 2.2, in this case, the phases $\delta$ and $\phi_{\mu, \tau}$ do not appear separately in the expression of the survival probability, see eq. (2.22). Therefore, it is enough to consider in our calculations the effective phase $\left(\delta-\phi_{\mu, \tau}\right)$.

The results corresponding to this particular case are shown in figure 6 . Here again we can see how the regions presented in the left panel of the figure agree with the behavior 

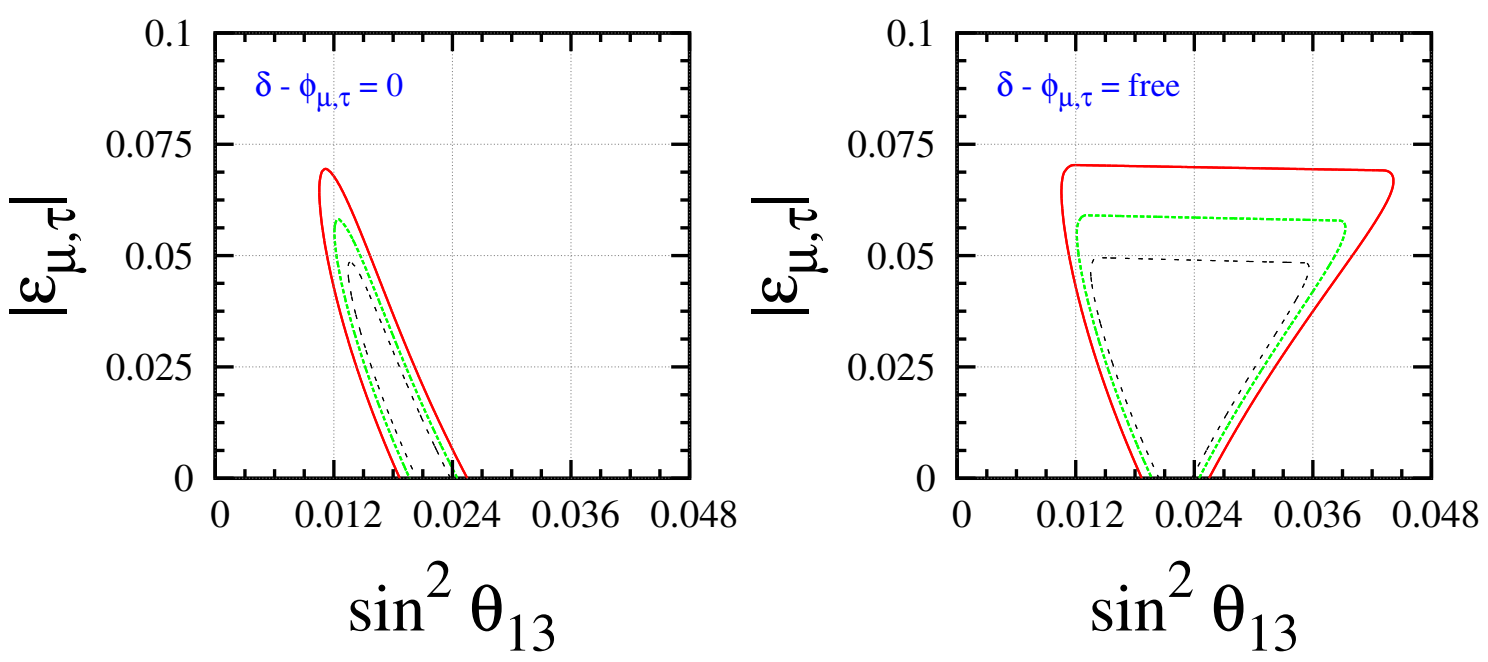

Figure 6. Allowed region in the $\sin ^{2} \theta_{13}-\left|\varepsilon_{\mu, \tau}\right|$ plane. Left panel is obtained setting the relevant phase $\left(\delta-\phi_{\mu, \tau}\right)$ to zero, while in the right panel $\left(\delta-\phi_{\mu, \tau}\right)$ is marginalized, varying freely between $-180^{\circ}$ and $180^{\circ}$. The regions correspond to $68 \%$ (black dashed line), $90 \%$ (green line), and $99 \%$ C.L. (red line) for 2 d.o.f.

shown in the iso-probability plots (right panel of figure 3) where there is an anticorrelation between the reactor angle and the NSI coupling $\left|\varepsilon_{\mu, \tau}\right|$. Thus, an increase in $\left|\varepsilon_{\mu, \tau}\right|$ is compensated by a shift of the preferred value of the reactor mixing angle toward smaller values, differently to what happens with the NSI coupling $\left|\varepsilon_{e}\right|$ in figure 5. The allowed interval for $\theta_{13}$ in this case is given by:

$$
0.013 \leq \sin ^{2} \theta_{13} \leq 0.024 \quad \text { (90\% C.L.) },
$$

while the obtained bound for the NSI coupling is the following

$$
\left|\varepsilon_{\mu, \tau}\right| \leq 5.1 \times 10^{-2} \quad(90 \% \text { C.L. }) .
$$

In this case reactor data can not improve the present constraints on the NSI couplings at eq. (1.3), and we get a limit of the same order of magnitude of the ones derived at ref. [49]. However, in both cases the limits have been derived using different data and assumptions, and therefore, they can be regarded as complementary bounds coming from different data sets.

In the right panel of figure 6 we show the results obtained when the phase $\left(\delta-\phi_{\mu, \tau}\right)$ is allowed to vary. In this case, a wider range in the reactor mixing angle is allowed:

$$
0.013 \leq \sin ^{2} \theta_{13} \leq 0.036 \quad(90 \% \text { C.L. }) .
$$

The reason is that, in addition to the anticorrelation shown in the left panel, a correlation between the reactor angle and $\left|\varepsilon_{\mu, \tau}\right|$ is also possible when the cosine function in eq. (2.23) is negative. Note, however, that both correlations are not symmetric, what results in the asymmetric behaviour of the allowed $\sin ^{2} \theta_{13}$ region with a bigger enlargement in the direction of increasing $\theta_{13}$. This can be explained by the presence of a linear term in $\sin \theta_{13}$ 

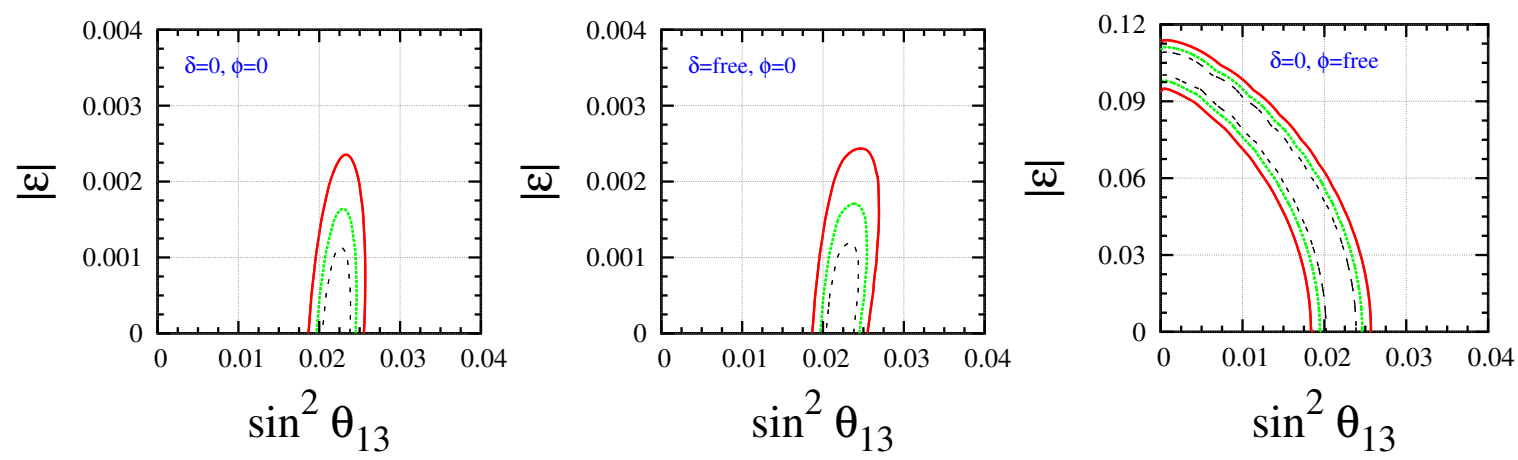

Figure 7. Allowed region in the $\sin ^{2} \theta_{13}-|\varepsilon|$ plane for different assumptions concerning the phases $\delta$ and $\phi$. The left panel is obtained switching all phases to zero, whereas in the middle panel $\phi=0$ and $\delta$ is left free. In the right panel $\delta$ is taken equal to zero while $\phi$ has been marginalized. The conventions for the lines is the same as in figure 5

in the redefinition of the effective reactor angle in the presence of NSI given in eq. (2.23). Nevertheless, even though there is a wider allowed region in the reactor angle, the bound on the $\left|\varepsilon_{\mu, \tau}\right|$ NSI coupling is nearly the same as the one obtained when the phase $\left(\delta-\phi_{\mu, \tau}\right)$ is set to zero, namely:

$$
\left|\varepsilon_{\mu, \tau}\right| \leq 5.2 \times 10^{-2} \quad \text { (90\% C.L.) } .
$$

\subsection{Constraints for the flavor-universal NSI case}

Here we present the results obtained under the hypothesis of flavor-universal NSI, that is, we assume all NSI couplings are present and they take the same value. Therefore, we consider only two NSI parameters: $|\varepsilon|$ and $\phi$, in addition to the standard model parameters entering in the calculations. In this case, the effective survival probability is given by the expression in eq. (2.24), with separate dependence on the phases $\delta$ and $\phi$. Therefore we have considered four different cases in our analysis: one with all the phases set to zero, two cases varying only one of the phases with the other set to zero and a last case varying the two phases simultaneously. Our results are presented at figure 7 .

The left panel in figure 7 shows the tight constraint obtained for the magnitude of the flavor-universal NSI coupling $|\varepsilon|$ when the phases are set to zero:

$$
|\varepsilon| \leq 1.2 \times 10^{-3} \quad(90 \% \text { C.L. }) .
$$

This result follows directly from the tendency already observed for $\left|\varepsilon_{e}\right|$ at figure 5. This happens because, even though the NSI couplings $\left|\varepsilon_{\mu, \tau}\right|$ are also present in the flavor-universal case, the dominant contribution comes from the non-oscillatory term depending on $\left|\varepsilon_{e}\right|$. The same behavior is also present in the middle panel of figure 7 , where the Dirac phase $\delta$ is allowed to freely vary. In this case, the effect of the Dirac phase is just a scaling in the last term in eq. (2.25), driven by $\cos \delta$, but again the total effect on $\theta_{13}$ is dominated by the non-oscillatory term in eq. (2.25). Under this assumption, we obtain the following bound on the magnitude of the flavor-universal coupling:

$$
|\varepsilon| \leq 1.3 \times 10^{-3} \quad(90 \% \text { C.L. }) .
$$


Note that in the two former cases the allowed range for the mixing angle $\theta_{13}$ is very close to the one obtained in the standard case, and therefore the Daya Bay determination is barely affected by the presence of NSI. However, when $\delta=0$ and the NSI phase $\phi$ is allowed to take different values, a completely different behavior results, as it is shown in the right panel of figure 7. In this case, in the fit of Daya Bay data, the phase $\phi$ takes a value such that the non-oscillatory term in eq. (2.24) is cancelled up to order $|\varepsilon|^{4}$. Most importantly, with a preferred value of $\cos \phi \simeq-1.5|\varepsilon|$, new terms of second order in $|\varepsilon|$ appear at eq. (2.24) and therefore the first order expression in eq. (2.25) can not satisfactory explain the degeneracy between $\theta_{13}$ and $|\varepsilon|$. Actually, the shift in the effective reactor angle is given now by this other expression (up to order $|\varepsilon|^{2}$ ):

$$
\tilde{s}_{13}^{2} \approx s_{13}^{2}+|\varepsilon|^{2}\left(2-3 \sqrt{2} s_{13}\right) \text {. }
$$

Then, it is possible to see that, even with $s_{13}=0$, one can reproduce the measured value of $\theta_{13}$ in Daya Bay with $|\varepsilon| \sim 0.11$, as shown in the corresponding plot. As a consequence of the degeneracy in the plane $s_{13}^{2}-|\varepsilon|$, this specific case is not very restrictive and we get a loose bound on $|\varepsilon|$ :

$$
|\varepsilon| \leq 1.1 \times 10^{-1} \quad(90 \% \text { C.L. }) .
$$

and only an upper bound on $\theta_{13}$ :

$$
\sin ^{2} \theta_{13} \leq 0.024 \quad(90 \% \text { C.L. })
$$

As commented above, the presence of flavor universal NSI implies that the reactor mixing angle may be compatible with zero. Nevertheless, the degeneracy between the mixing angle and the new physics parameter $|\varepsilon|$ observed here may be lifted by a combined analysis with accelerator long-baseline neutrino experiments. A global analysis of neutrino data assuming the simultaneous presence of NSI in reactor and accelerator neutrino data would be very useful for this purpose. Besides solving the degeneracy, the combined analysis might provide further constraints on the NSI couplings as well as improve the agreement between the preferred $\theta_{13}$ value from reactors and long-baseline experiments, as discussed in ref. [80-82]. However, since the production, detection and propagation of neutrinos is quite different in both kind of experiments, a global analysis would require a very detailed study with many new physics parameters involved, besides the consideration of a specific model for NSI. In any case, this point is out of the scope of the present analysis and it will be considered elsewhere.

Finally, let us comment that we have also considered the case of flavor-universal NSI with all the phases different from zero. However, we have not presented the results obtained for this setup because, in this case, the confusion between $\theta_{13}$ and $|\varepsilon|$ is complete, and therefore no information on any of the parameters can be extracted from the analysis of Daya Bay data. All the results obtained in this section are summarized in table 2. Needless to mention, to obtain all the limits on $\sin ^{2} \theta_{13}$ presented along this section as well as in the table, we have marginalized over the NSI couplings over a wide range. Similarly, to place bounds on the NSI parameters, $\sin ^{2} \theta_{13}$ has also been allowed to float over a wide range. 


\begin{tabular}{|l|c|c|}
\hline phases & $\sin ^{2} \theta_{13}$ & $|\varepsilon|$ \\
\hline \multicolumn{3}{|c|}{ electron-type NSI coupling } \\
\hline$\phi_{e}=0$ & $0.020 \leq \sin ^{2} \theta_{13} \leq 0.024$ & $\left|\varepsilon_{e}\right| \leq 0.0012$ \\
$\phi_{e}$ free & $0.020 \leq \sin ^{2} \theta_{13} \leq 0.024$ & $\left|\varepsilon_{e}\right|$ unbound \\
\hline \multicolumn{3}{|c|}{ muon or tau-type NSI couplings } \\
\hline$\left(\delta-\phi_{\mu, \tau}\right)=0$ & $0.013 \leq \sin ^{2} \theta_{13} \leq 0.024$ & $\left|\varepsilon_{\mu, \tau}\right| \leq 0.051$ \\
$\left(\delta-\phi_{\mu, \tau}\right)$ free & $0.013 \leq \sin ^{2} \theta_{13} \leq 0.036$ & $\left|\varepsilon_{\mu, \tau}\right| \leq 0.052$ \\
\hline \multicolumn{3}{|c|}{ universal NSI couplings } \\
\hline$\delta=\phi=0$ & $0.020 \leq \sin ^{2} \theta_{13} \leq 0.024$ & $|\varepsilon| \leq 0.0012$ \\
\hline$\delta$ free, $\phi=0$ & $0.020 \leq \sin ^{2} \theta_{13} \leq 0.025$ & $|\varepsilon| \leq 0.0013$ \\
\hline$\delta=0, \phi$ free & $\sin ^{2} \theta_{13} \leq 0.024$ & $|\varepsilon| \leq 0.110$ \\
\hline
\end{tabular}

Table 2. $90 \%$ C.L. bounds ( 1 d.o.f) on $\sin ^{2} \theta_{13}$ and the NSI couplings from current Daya Bay data without considering any uncertainty in the normalization of reactor event rates in the statistical analysis $\left(a_{\text {norm }}=0\right)$.

\section{Bounds on NSI from Daya Bay with $5 \%$ normalization error}

In the previous section, we have not considered any normalization error in the statistical analysis of Daya Bay reactor data. This means that we have assumed a perfect knowledge of the event normalization at the experiment, disregarding the presence of uncertainties in the flux reactor normalization or in the detection cross section, among others. This procedure has been followed in most of the previous phenomenological analyses of Daya Bay data in presence of NSI, see for instance ref. [47]. In the more recent work at ref. [48], the authors have considered small uncertainties in the reactor flux and in the detector properties, although they did not take into account an uncertainty in the overall normalization of the event rates. Needless to say that a more detailed analysis of reactor data can not ignore the presence of such normalization errors. Therefore, in this section we present a $\chi^{2}$ analysis of Daya Bay data using the expression defined at eq. (3.2), where a free normalization factor is considered in order to account for the uncertainties in the total event number normalization. This point is very relevant in the study of NSI with reactor experiments, since the uncertainty in the event normalization presents a degeneracy with the zerodistance effect due to NSI. In consequence, the far over near technique exploited by Daya Bay in order to reduce the dependence upon total normalization does not work equally fine in the presence of NSI, where the non-oscillatory zero-distance effect, simultaneously present at near and far detectors, does not totally cancel. Actually, in the standard model case without NSI, the number of events expected at the near detector is given by:

$$
N_{\mathrm{ND}}^{\mathrm{SM}} \simeq N\left(1+a_{\mathrm{norm}}\right) P_{e e}^{\mathrm{SM}}(L=0)=N\left(1+a_{\mathrm{norm}}\right)
$$


while, in the presence of NSI, the event number at the near detector is calculated as follows:

$$
\begin{aligned}
N_{\mathrm{ND}}^{\mathrm{NSI}} & \simeq N\left(1+a_{\mathrm{norm}}\right) P_{e e}^{\mathrm{NSI}}(L=0)=N\left(1+a_{\mathrm{norm}}\right)(1+f(\varepsilon)) \\
& \simeq N\left(1+f(\varepsilon)+a_{\text {norm }}\right) .
\end{aligned}
$$

Here $a_{\text {norm }}$ controls the normalization of far and near detector events in the fit and, together with the NSI couplings $\varepsilon$, it fixes the total zero-distance effect. As a result, if we set $a_{\text {norm }}$ to zero, we artificially increase the power of Daya Bay data to constrain the zero-distance effect due to NSI, getting non-realistic strong bounds on the NSI couplings. On the other hand, we can not leave the factor $a_{\text {norm }}$ totally free in our statistical analysis, as it is usually done in the standard Daya Bay analysis, where the factor is kept small thanks to the far over near technique. Actually, we have found that leaving the normalization factor totally free, and due to the degeneracy with the NSI couplings, it could achieve very large values, of the order of $10-20 \%$. For this reason it is necessary the use of a prior on this magnitude. Recent reevaluations of the reactor antineutrino flux indicate an uncertainty on the total flux of about 3\% [77, 83]. However, an independent analysis in ref. [84] claims that this uncertainty may have been underestimated due to the treatment of forbidden transitions in the antineutrino flux evaluation, and proposes a total uncertainty of $4 \%$. Since the total normalization errors may also include uncertainties coming from other sources, we follow the conservative approach of taking a total uncertainty on the reactor event normalization of $5 \%$. This is the value we have assumed for $\sigma_{a}$ in eq. (3.2).

To illustrate the differences with respect to the results obtained in the previous section, assuming no uncertainties in the event rate normalization, here we have considered only the cases where all phases are set to zero. ${ }^{3}$ The results obtained with these assumptions are presented in figure 8 and table 3 . In the left panel of figure 8 we present the allowed region in the plane $\sin ^{2} \theta_{13}-\left|\varepsilon_{e}\right|$ when only NSI with electron antineutrinos are present. In this case, the range for the reactor mixing angle is rather similar to the one shown in the left panel of figure 5:

$$
0.020 \leq \sin ^{2} \theta_{13} \leq 0.025 \quad \text { (90\% C.L.) },
$$

while the bound on $\left|\varepsilon_{e}\right|$, however, is much weaker than the one given at eq. (4.1) (although still slightly better than the one at eq. (1.3)):

$$
\left|\varepsilon_{e}\right| \leq 1.5 \times 10^{-2} \quad(90 \% \text { C.L. }) \text {. }
$$

The same bound is also obtained in the flavor-universal case for the NSI parameter $|\varepsilon|$, see the right panel of figure 8 . In this case, the allowed range for the reactor mixing angle is a bit enlarged with respect to the previous one:

$$
0.017 \leq \sin ^{2} \theta_{13} \leq 0.024 \quad(90 \% \text { C.L. }),
$$

due to the presence of NSI oscillation terms driven by the new physics couplings with muon and tau antineutrinos. As commented above, the loss of sensitivity to the NSI couplings is

\footnotetext{
${ }^{3}$ Note that, in this case, the correction terms to the effective reactor angle at first order in $|\varepsilon|$ dominate and, therefore, second order corrections are not relevant.
} 

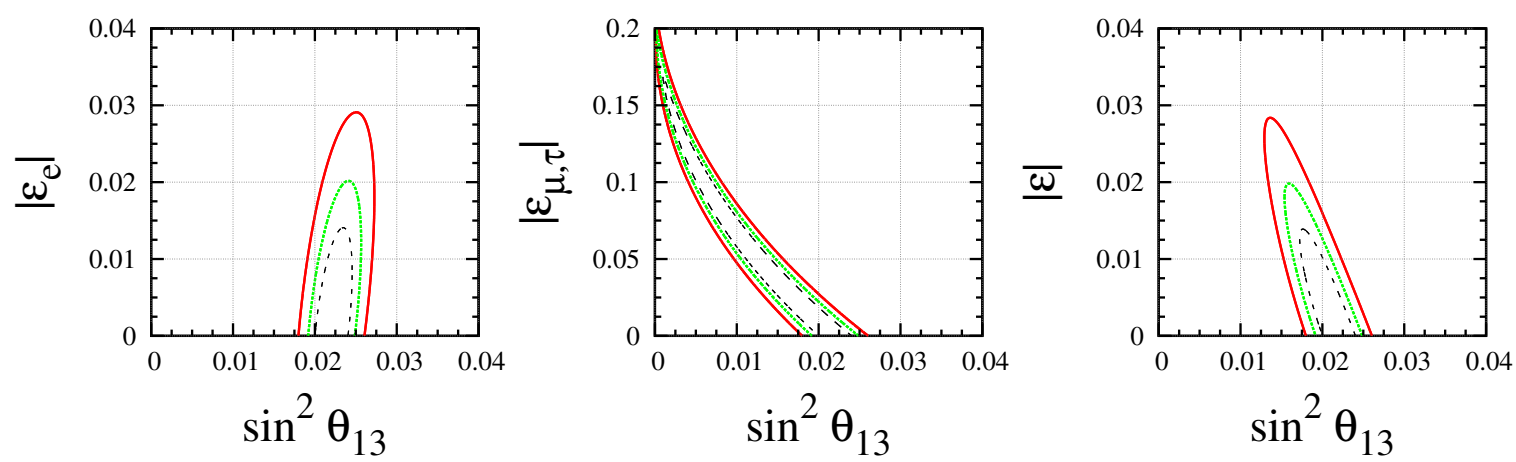

Figure 8. Allowed regions in the $\sin ^{2} \theta_{13}$ - NSI coupling plane for the different cases considered: $\left|\varepsilon_{e}\right|$ (left panel), $\left|\varepsilon_{\mu, \tau}\right|$ (middle panel) and $|\varepsilon|$ (right panel) setting all the phases equal to zero and assuming $5 \%$ uncertainty on the total event rate normalization of Daya Bay events. The conventions for the lines is the same as in figure 5 .

\begin{tabular}{|l|c|c|}
\hline Case & $\sin ^{2} \theta_{13}$ & $|\varepsilon|$ \\
\hline$\phi_{e}=0$ & $0.020 \leq \sin ^{2} \theta_{13} \leq 0.025$ & $\left|\varepsilon_{e}\right| \leq 0.015$ \\
$\left(\delta-\phi_{\mu, \tau}\right)=0$ & $\sin ^{2} \theta_{13} \leq 0.024$ & $\left|\varepsilon_{\mu, \tau}\right| \leq 0.176$ \\
$\delta=\phi=0$ & $0.017 \leq \sin ^{2} \theta_{13} \leq 0.024$ & $|\varepsilon| \leq 0.015$ \\
\hline
\end{tabular}

Table 3. $90 \%$ C.L. bounds ( 1 d.o.f) on $\sin ^{2} \theta_{13}$ and the NSI couplings using current Daya Bay data with a $5 \%$ uncertainty on the total event normalization.

due to the degeneracy between the normalization uncertainty and the zero-distance terms induced by the presence of NSI. In this way, a larger value of the NSI parameters can be compensated with a non-zero normalization factor $a_{\text {norm }}$, without spoiling the good agreement with experimental reactor data.

Finally, the middle panel of figure 8 shows the results obtained when only NSI with muon or tau antineutrinos are considered. In this case, the cancellation between the normalization term and the zero-distance effect due to terms of second order in $\left|\varepsilon_{\mu, \tau}\right|$ results in an extended region in the $\sin ^{2} \theta_{13}-\left|\varepsilon_{\mu, \tau}\right|$ plane with an upper bound of:

$$
\sin ^{2} \theta_{13} \leq 0.024 \quad \text { (90\% C.L.) } .
$$

The bound on the NSI coupling is given by:

$$
\left|\varepsilon_{\mu, \tau}\right| \leq 0.176 \quad \text { (90\% C.L.) } .
$$

\section{Comparing NSI constraints from 217 and 621 days of Daya Bay run}

The new high-precision data from Daya Bay with 621 days of running time [3] has improved the measurement of $\sin ^{2} 2 \theta_{13}$ dramatically with a relative $1 \sigma$ precision of $\sim 6 \%$ as compared to $\sim 11 \%$ obtained using the previously published 217 days of data [2]. This clearly shows the impact of the four time more statistics that the Daya Bay experiment has accumulated 

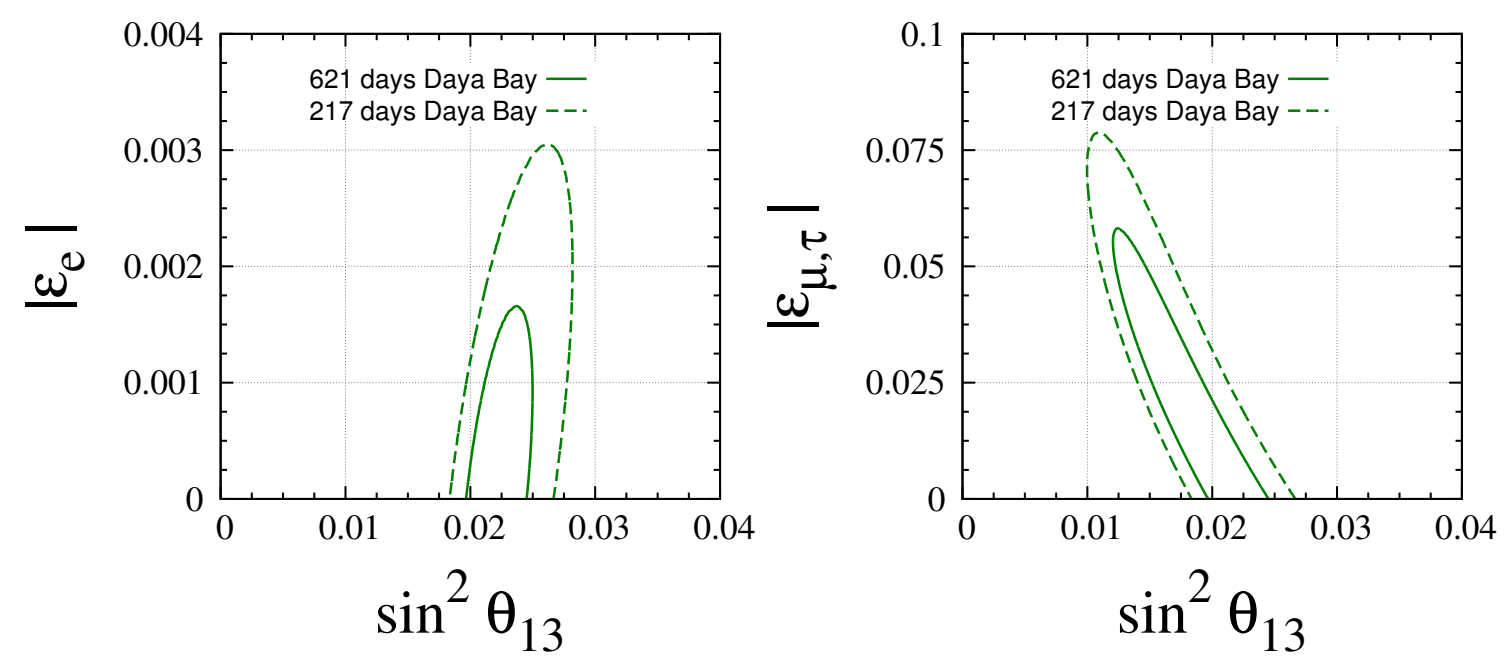

Figure 9. Left panel shows the allowed parameter space in $\sin ^{2} \theta_{13}-\varepsilon_{e} \mid$ plane at 90\% C.L. (2 d.o.f). Right panel depicts the same in $\sin ^{2} \theta_{13}-\left|\varepsilon_{\mu, \tau}\right|$ plane. Here the solid (dashed) lines correspond to the new (old) 621 (217) days of Daya Bay data. Here all the phases are considered to be zero and the normalization of events is fixed in the statistical analysis with $a_{\text {norm }}=0$.

with the help of eight ADs in comparison with the previously released Daya Bay data set with six ADs. Now it would be quite interesting to see how much we can further constrain the allowed ranges for these NSI parameters under consideration using the new 621 days of Daya Bay data in comparison with the old 217 days of Daya Bay run. In figure 9, we compare the performance of the current and the previous data sets of Daya Bay in constraining the allowed regions in $\sin ^{2} \theta_{13}-\left|\varepsilon_{e}\right|$ plane (left panel) and in $\sin ^{2} \theta_{13}-\left|\varepsilon_{\mu, \tau}\right|$ plane (right panel). In both the panels, the solid (dashed) lines portray the results with the new (old) 621 (217) days of Daya Bay data. For the sake of illustration, we have only chosen the cases of NSI parameters which are associated with $\bar{\nu}_{e}$ (left panel) and $\bar{\nu}_{\mu}$ (right panel) assuming all the phases to be zero. In particular, we have focused on the situations which are presented in sections 4.1 and 4.2, where we do not consider the normalization uncertainty on the reactor events and set $a_{\text {norm }}=0$ in the statistical analysis. As compared to the old data set, with the current data, the improvement in constraining the allowed parameter space between $\sin ^{2} \theta_{13}$ and the NSI parameters is quite significant as can be readily seen from figure 9 .

In table 4 , we present the $90 \%$ C.L. ( 1 d.o.f) constraints on $\sin ^{2} \theta_{13}$ and the NSI parameters using the previous (current) 217 (621) days of Daya Bay data. Here all the phases are considered to be zero. We do not consider the normalization uncertainty on the reactor events and take $a_{\text {norm }}=0$ in the statistical analysis. We can see from table 4 that in case of electron-type and universal NSI parameters, the bounds on $\left|\varepsilon_{e}\right|$ and $|\varepsilon|$ are the same while analyzing 621 days of Daya Bay data. This feature is also there in the case of 217 days of Daya Bay run. The constraints on $\left|\varepsilon_{e}\right|$ and $|\varepsilon|$ get improved by factor of two when we consider the current 621 days of Daya Bay data compared to its previous 217 days of data. We also get better bounds on $\left|\varepsilon_{\mu, \tau}\right|$ using the current Daya Bay data as compared to the old data set. These results suggest that the future data from the Daya Bay 


\begin{tabular}{|l|c|c|}
\hline Daya Bay data & $\sin ^{2} \theta_{13}$ & $|\varepsilon|$ \\
\hline \multicolumn{3}{|c|}{ electron-type NSI parameters } \\
\hline Current (621 days) & $0.020 \leq \sin ^{2} \theta_{13} \leq 0.024$ & $\left|\varepsilon_{e}\right| \leq 0.0012$ \\
Previous (217 days) & $0.019 \leq \sin ^{2} \theta_{13} \leq 0.027$ & $\left|\varepsilon_{e}\right| \leq 0.0024$ \\
\hline \multicolumn{3}{|c|}{ muon or tau-type NSI parameters } \\
\hline Current (621 days) & $0.013 \leq \sin ^{2} \theta_{13} \leq 0.024$ & $\left|\varepsilon_{\mu, \tau}\right| \leq 0.051$ \\
Previous (217 days) & $0.011 \leq \sin ^{2} \theta_{13} \leq 0.026$ & $\left|\varepsilon_{\mu, \tau}\right| \leq 0.070$ \\
\hline \multicolumn{3}{|c|}{ universal NSI parameters } \\
\hline Current (621 days) & $0.020 \leq \sin ^{2} \theta_{13} \leq 0.024$ & $|\varepsilon| \leq 0.0012$ \\
Previous (217 days) & $0.019 \leq \sin ^{2} \theta_{13} \leq 0.026$ & $|\varepsilon| \leq 0.0024$ \\
\hline
\end{tabular}

Table 4. $90 \%$ C.L. (1 d.o.f) bounds on $\sin ^{2} \theta_{13}$ and the NSI parameters using the old (new) 217 (621) days of Daya Bay data. Here all the phases are considered to be zero. We also do not consider the uncertainty on the normalization of reactor events and set $a_{\text {norm }}=0$ in the statistical analysis.

\begin{tabular}{|c|c|c|}
\hline Daya Bay data & $\sin ^{2} \theta_{13}$ & $|\varepsilon|$ \\
\hline \multicolumn{2}{|c|}{ electron-type NSI parameters $\left[\phi_{e}\right.$ free $]$} \\
\hline Current (621 days) & $0.020 \leq \sin ^{2} \theta_{13} \leq 0.024$ & $\left|\varepsilon_{e}\right|$ unbound \\
Previous (217 days) & $0.019 \leq \sin ^{2} \theta_{13} \leq 0.027$ & $\left|\varepsilon_{e}\right|$ unbound \\
\hline \multicolumn{3}{|c|}{ muon or tau-type NSI parameters $\left[\left(\delta-\phi_{\mu, \tau}\right)\right.$ free $]$} \\
\hline Current (621 days) & $0.013 \leq \sin ^{2} \theta_{13} \leq 0.036$ & $\left|\varepsilon_{\mu, \tau}\right| \leq 0.052$ \\
Previous (217 days) & $0.011 \leq \sin ^{2} \theta_{13} \leq 0.045$ & $\left|\varepsilon_{\mu, \tau}\right| \leq 0.070$ \\
\hline \multicolumn{3}{|c|}{ universal NSI parameters $[\delta$ free, $\phi=0]$} \\
\hline Current (621 days) & $0.020 \leq \sin ^{2} \theta_{13} \leq 0.025$ & $|\varepsilon| \leq 0.0013$ \\
Previous (217 days) & $0.019 \leq \sin ^{2} \theta_{13} \leq 0.028$ & $|\varepsilon| \leq 0.0024$ \\
\hline \multicolumn{3}{|c|}{ universal NSI parameters $[\delta=0, \phi$ free] } \\
\hline Current (621 days) & $\sin ^{2} \theta_{13} \leq 0.024$ & $|\varepsilon| \leq 0.110$ \\
Previous (217 days) & $\sin ^{2} \theta_{13} \leq 0.026$ & $|\varepsilon| \leq 0.116$ \\
\hline
\end{tabular}

Table 5. $90 \%$ C.L. (1 d.o.f) bounds on $\sin ^{2} \theta_{13}$ and the NSI parameters using the old (new) 217 (621) days of Daya Bay data. Here we allow the phases or their certain combinations to vary freely. We do not consider the uncertainty on the normalization of reactor events and set $a_{\text {norm }}=0$ in the statistical analysis.

experiment with more statistics is going to play an important role to further constrain the allowed parameter space for the NSI parameters. There are also marginal improvements on the bounds to $\sin ^{2} \theta_{13}$ with the current 621 days of Daya Bay data.

Finally in table 5, we compare the the $90 \%$ C.L. (1 d.o.f) limits on $\sin ^{2} \theta_{13}$ and the NSI parameters obtained using the old 217 days and new 621 days of Daya Bay data allowing 
the phases or their certain combinations to vary freely as we consider in table 2 in section 4 . Like in table 4 , here also we do not take into account the normalization uncertainty on the reactor events and consider $a_{\text {norm }}=0$ in the statistical analysis. Table 5 depicts that even if we allow the phases to vary freely, we obtain better limits on the NSI parameters with the new data set as compared to the previous 217 days of Daya Bay data, except for the case of $\left|\varepsilon_{e}\right|$ which remains unbounded. Table 5 indicates that the new data set of Daya Bay also reduces the allowed ranges for $\sin ^{2} \theta_{13}$ in the presence of NSI parameters as compared to the old data. This improvement is quite significant in the case of $\left|\varepsilon_{\mu, \tau}\right|$ even if the effective phase $\left(\delta-\phi_{\mu, \tau}\right)$ is allowed to vary freely in the fit.

\section{Summary and conclusions}

The success of the currently running Daya Bay, RENO, and Double Chooz reactor antineutrino experiments in measuring the smallest lepton mixing angle $\theta_{13}$ with impressive accuracy signifies an important advancement in the field of modern neutrino physics with nonzero mass and three-flavor mixing. With this remarkable discovery, the neutrino oscillation physics has entered into a high-precision era opening up the possibility of observing sub-dominant effects due to possible new physics beyond the Standard Model of particle physics. At present, undoubtedly the Daya Bay experiment in China is playing a leading and an important role in this direction. The recent high-precision and unprecedentedly copious data from the Daya Bay experiment has provided us an opportunity to probe the existence of the non-standard interaction effects which might crop up at the production point or at the detection stage of the reactor antineutrinos.

In this paper for the first time, we have reported the new constraints on the flavor nonuniversal and also flavor universal NSI parameters obtained using the currently released 621 days of Daya Bay data. While placing the bounds on these NSI parameters, we have assumed that the new physics effects are just inverse of each other in the production and detection processes of the reactor antineutrino experiment ,i.e., $\varepsilon_{e \gamma}^{s}=\varepsilon_{\gamma e}^{d *}$. Considering this special case, we have discussed in detail the impact of the NSI parameters on the effective antineutrino survival probability expressions which we ultimately use to analyze the Daya Bay data. With this special choice of the NSI parameters, we have observed a shift in the oscillation amplitude without altering the $L / E$ pattern of the oscillation probability. This shift in the depth of the oscillation dip can be caused due to the NSI parameters and as well as $\theta_{13}$, making it quite difficult to disentangle the NSI effects from the standard oscillations. Before presenting the final results, we have studied the correlations between the NSI parameters and $\theta_{13}$ with the help of iso-probability surface plots in section 2 . This study has been quite useful to understand the final bounds on the NSI parameters that we have obtained from the fit. Since the shape of the oscillation probability is not distorted with the special choice of the NSI parameters considered in this paper, an analysis based on the total event rate at the Daya Bay experiment is sufficient to obtain the limits on the NSI parameters.

As far as the flavor non-universal NSI parameters are concerned, first we have considered the NSI parameters $\left|\varepsilon_{e}\right|$ and $\phi_{e}$ which are associated with $\bar{\nu}_{e}$. Assuming $\phi_{e}=0^{\circ}$ and a perfect knowledge of the normalization of the event rates, the current Daya Bay data 
places a strong constrain on $\left|\varepsilon_{e}\right| \leq 1.2 \times 10^{-3}$ (90\% C.L.) improving the present bound on $\left|\varepsilon_{e}\right|$ by one order of magnitude. Now if we consider the uncertainty in the normalization of event rates with a prior of $5 \%$, then this limit changes to $15 \times 10^{-3}$, diluting the constraint by almost one order of magnitude. No limits can be placed on $\left|\varepsilon_{e}\right|$ if we allow $\phi_{e}$ to vary freely in the fit. We have also observed that the determination of the 1-3 mixing angle is quite robust in this specific case and it is almost independent of the issue of uncertainty in the normalization of event rates and the choice of $\phi_{e}$. In fact, the allowed range of $\sin ^{2} \theta_{13}$ coincides exactly with the allowed range in absence of NSI. Next we turn our attention to the NSI parameters $\left|\varepsilon_{\mu}\right|$ and $\phi_{\mu}$ which are associated with $\bar{\nu}_{\mu}$. With $\left(\delta-\phi_{\mu}\right)=0^{\circ}$ and a perfect knowledge of the event rates normalization, the current Daya Bay data sets a limit of $\left|\varepsilon_{\mu}\right| \leq 5.1 \times 10^{-2}$ (90\% C.L.) which is comparable and complementary to the existing bound obtained using a different data set under different assumptions. This limit on $\left|\varepsilon_{\mu}\right|$ becomes $17.6 \times 10^{-2}$ once we consider the uncertainty in the normalization of event rates with a prior of $5 \%$. These limits on $\left|\varepsilon_{\mu}\right|$ remain almost unchanged even if we allow $\left(\delta-\phi_{\mu}\right)$ to vary freely in the fit. This is not true while placing the constraint on $\sin ^{2} \theta_{13}$. For an example, in the case when we do not consider any uncertainty in the normalization of event rates, the upper bound on $\sin ^{2} \theta_{13}$ increases from $2.4 \times 10^{-2}$ to $3.6 \times 10^{-2}$ due to freely varying $\left(\delta-\phi_{\mu}\right)$ in the fit instead of setting it to zero. We cannot place a lower bound on $\sin ^{2} \theta_{13}$ when we consider the uncertainty in the normalization of event rates with a prior of $5 \%$ even if $\left(\delta-\phi_{\mu}\right)$ is taken to be zero in the fit. The above mentioned limits on $\left|\varepsilon_{\mu}\right|$ and $\sin ^{2} \theta_{13}$ are also valid for the NSI parameters $\left|\varepsilon_{\tau}\right|$ and $\phi_{\tau}$ as long as the 2-3 mixing angle is maximal ,i.e., $\sin ^{2} \theta_{23}=0.5$.

In the case of flavor universal NSI parameters, we have placed limits on $|\varepsilon|$ and $\sin ^{2} \theta_{13}$ under certain assumptions on $\delta$ and $\phi$. With $\delta=\phi=0^{\circ}$ and perfect knowledge of the normalization of the event rates, the upper bound on $|\varepsilon|$ at $90 \%$ C.L. ( 1 d.o.f) is $1.2 \times 10^{-3}$. Though this limit does not change much when we marginalize over $\delta$ in the fit, it deteriorates by almost ninety times when we allow $\phi$ to vary freely in the fit providing a limit of $|\varepsilon| \leq 110 \times 10^{-3}(90 \%$ C.L.). With a $5 \%$ uncertainty in the normalization of total event rates and $\delta=\phi=0^{\circ}$, the constraint on $|\varepsilon|$ becomes $\leq 15 \times 10^{-3}$ at $90 \%$ C.L. With the same assumptions, we can restrict $\theta_{13}$ within a range of $0.017 \leq \sin ^{2} \theta_{13} \leq 0.024$ (90\% C.L.).

One of the novelties of this work is the inclusion of correction terms of second order in the NSI couplings $|\varepsilon|$ in the effective neutrino probability in eq. (2.17). The role of these second order corrections at the effective probability level has been analyzed in section 2.2 as well as in the discussion of the probability and correlation plots in section 2.3 and section 2.4. The impact of second order terms on the determination of $\sin ^{2} \theta_{13}$ is specially relevant for the flavour-universal-NSI case, as commented in section 4.3.

One of the interesting studies that we have performed in this paper is the comparison of the constraints on the NSI parameters placed with the current 621 days of Daya Bay data with the limits obtained using the previously released 217 days of Daya Bay run. In this analysis, all the phases are considered to be zero and the normalization of events is also kept fixed in the statistical analysis with $a_{\text {norm }}=0$. We have observed that the constraints on $\left|\varepsilon_{e}\right|$ and $|\varepsilon|$ get improved by factor of two when we analyze the current 621 days of Daya Bay data compared to its previous 217 days data. This comparative study reveals the merit of the huge statistics that Daya Bay has already accumulated. It also suggests that the future 
high-precision data from the Daya Bay experiment with enhanced statistics is inevitable to further probe the sub-leading effects in neutrino flavor conversion due to the presence of the possible NSI parameters beyond the standard three-flavor oscillation paradigm.

\section{Acknowledgments}

S.K.A. acknowledges the support from DST/INSPIRE Research Grant [IFA-PH-12], Department of Science and Technology, India. The work of D.V.F. and M.T. was supported by the Spanish grants FPA2011-22975 and MULTIDARK CSD2009-00064 (MINECO) and PROMETEOII/2014/084 (Generalitat Valenciana). This work has also been supported by the U.S. Department of Energy under award number DE-SC0003915.

\section{A Effective survival probability with the NSI parameters: $\varepsilon_{e \gamma}^{s} \neq \varepsilon_{\gamma e}^{d *}$}

In this appendix, we present the effective probability expressions for the physical scenarios where $\varepsilon_{e \gamma}^{s} \neq \varepsilon_{\gamma e}^{d *}$. In such cases, the spectral study of the reactor data plays an important role since the NSI parameters are not only responsible for a shift in $\theta_{13}$ i.e. the change of the depth of the first oscillation maximum but they also modify the $L / E$ pattern of the probability due to the shift in its energy. We have already mentioned that a detailed analysis of the Daya Bay data considering such interesting physical cases will be performed in [69].

\section{A.1 Presence of the NSI parameters only at the production stage}

Here we assume that the NSI parameters only affect the production mechanism of the antineutrinos in the reactor experiment. It allows us to write (dropping the universal e-index):

$$
\varepsilon_{\gamma}^{s}=\left|\varepsilon_{\gamma}\right| \mathrm{e}^{\mathrm{i} \phi_{\gamma}}, \text { and } \varepsilon_{\gamma}^{d}=0 .
$$

With this assumption, we get the effective neutrino survival probability as follows:

$$
\begin{aligned}
P_{\bar{\nu}_{e}^{s} \rightarrow \bar{\nu}_{e}^{d}} \simeq & P_{\bar{\nu}_{e}^{s} \rightarrow \bar{\nu}_{e}^{d}}^{\mathrm{SM}}+\left|\varepsilon_{e}\right|^{2}+2\left|\varepsilon_{e}\right| \cos \phi_{e} \\
& +2 s_{13}\left[s_{23}\left|\varepsilon_{\mu}\right| \sin \left(\delta-\phi_{\mu}\right)+c_{23}\left|\varepsilon_{\tau}\right| \sin \left(\delta-\phi_{\tau}\right)\right] \sin \left(2 \Delta_{31}\right) \\
& -4 s_{13}\left[s_{23}\left|\varepsilon_{\mu}\right| \cos \left(\delta-\phi_{\mu}\right)+c_{23}\left|\varepsilon_{\tau}\right| \cos \left(\delta-\phi_{\tau}\right)\right] \sin ^{2}\left(\Delta_{31}\right) \\
& +\sin 2 \theta_{12}\left[-c_{23}\left|\varepsilon_{\mu}\right| \sin \phi_{\mu}+s_{23}\left|\varepsilon_{\tau}\right| \sin \phi_{\tau}\right] \sin \left(2 \Delta_{21}\right)
\end{aligned}
$$

\section{A.2 NSI at the source and detector with the same magnitude and different phases}

In this case, we assume that the magnitude of the NSI parameters is the same at the production and detection level, but the phases associated with the NSI parameters are different at the source and detector. Under this situation, we can write:

$$
\varepsilon_{\gamma}^{s}=\left|\varepsilon_{\gamma}\right| \mathrm{e}^{\mathrm{i} \phi_{\gamma}^{s}}, \text { and } \varepsilon_{\gamma}^{d}=\left|\varepsilon_{\gamma}\right| \mathrm{e}^{\mathrm{i} \phi_{\gamma}^{d}}
$$

Under this assumption, the effective neutrino survival probability takes the form:

$$
P_{\bar{\nu}_{e}^{s} \rightarrow \bar{\nu}_{e}^{d}} \simeq P_{\bar{\nu}_{e} \rightarrow \bar{\nu}_{e}}^{\mathrm{SM}}+P_{\text {non-osc }}^{\mathrm{NSI-IIb}}+P_{\mathrm{osc}-\mathrm{atm}}^{\mathrm{NSI}}+P_{\mathrm{osc}-\text { solar }}^{\mathrm{NSIIII}},
$$


where the non-standard terms are given by:

$$
\begin{aligned}
P_{\text {non-osc }}^{\text {NSI-IIb }}= & 2\left\{\left|\varepsilon_{e}\right|\left(\cos \phi_{e}^{d}+\cos \phi_{e}^{s}\right)+\left|\varepsilon_{e}\right|^{2}\left[1+\cos \left(\phi_{e}^{d}-\phi_{e}^{s}\right)+\cos \left(\phi_{e}^{d}+\phi_{e}^{s}\right)\right]\right. \\
& \left.+\left|\varepsilon_{\mu}\right|^{2} \cos \left(\phi_{\mu}^{s}+\phi_{\mu}^{d}\right)+\left|\varepsilon_{\tau}\right|^{2} \cos \left(\phi_{\tau}^{s}+\phi_{\tau}^{d}\right)\right\} \\
P_{\text {osc-atm }}^{\text {NSI-IIb }}= & 2\left\{s_{13} s_{23}\left|\varepsilon_{\mu}\right|\left[\sin \left(\delta-\phi_{\mu}^{s}\right)-\sin \left(\delta+\phi_{\mu}^{d}\right)\right]+s_{13} c_{23}\left|\varepsilon_{\tau}\right|\left[\sin \left(\delta-\phi_{\tau}^{s}\right)-\sin \left(\delta+\phi_{\tau}^{d}\right)\right]\right. \\
& -s_{23}^{2}\left|\varepsilon_{\mu}\right|^{2} \sin \left(\phi_{\mu}^{s}+\phi_{\mu}^{d}\right)-c_{23}^{2}\left|\varepsilon_{\tau}\right|^{2} \sin \left(\phi_{\tau}^{s}+\phi_{\tau}^{d}\right) \\
& \left.-c_{23} s_{23}\left|\varepsilon_{\mu}\right|\left|\varepsilon_{\tau}\right|\left[\sin \left(\phi_{\tau}^{s}+\phi_{\mu}^{d}\right)+\sin \left(\phi_{\mu}^{s}+\phi_{\tau}^{d}\right)\right]\right\} \sin \left(2 \Delta_{31}\right) \\
& -4\left\{s_{13} s_{23}\left|\varepsilon_{\mu}\right|\left[\cos \left(\delta-\phi_{\mu}^{s}\right)+\cos \left(\delta+\phi_{\mu}^{d}\right)\right]+c_{23} s_{13}\left|\varepsilon_{\tau}\right|\left[\cos \left(\delta-\phi_{\tau}^{s}\right)+\cos \left(\delta+\phi_{\tau}^{d}\right)\right]\right. \\
& +s_{23}^{2}\left|\varepsilon_{\mu}\right|^{2} \cos \left(\phi_{\mu}^{s}+\phi_{\mu}^{d}\right)+c_{23}^{2}\left|\varepsilon_{\tau}\right|^{2} \cos \left(\phi_{\tau}^{s}+\phi_{\tau}^{d}\right) \\
& \left.+c_{23} s_{23}\left|\varepsilon_{\mu}\right|\left|\varepsilon_{\tau}\right|\left[\cos \left(\phi_{\tau}^{s}+\phi_{\mu}^{d}\right)+\cos \left(\phi_{\mu}^{s}+\phi_{\tau}^{d}\right)\right]\right\} \sin ^{2}\left(\Delta_{31}\right), \\
P_{\text {osc-solar }}^{\text {NSIIIb }}= & \left.\sin 2 \theta_{12}\left[-c_{23}\left|\varepsilon_{\mu}\right|\left(\sin \phi_{\mu}^{s}+\sin \phi_{\mu}^{d}\right)+s_{23}\left|\varepsilon_{\tau}\right|\left(\sin \phi_{\tau}^{s}+\sin \phi_{\tau}^{d}\right)\right] \sin \left(2 \Delta_{21}\right) \cdot \text { (A. } .\right)
\end{aligned}
$$

Open Access. This article is distributed under the terms of the Creative Commons Attribution License (CC-BY 4.0), which permits any use, distribution and reproduction in any medium, provided the original author(s) and source are credited.

\section{References}

[1] Daya Bay collaboration, F.P. An et al., Improved measurement of electron antineutrino disappearance at Daya Bay, Chin. Phys. C 37 (2013) 011001 [arXiv:1210.6327] [InSPIRE].

[2] Daya Bay collaboration, F.P. An et al., Spectral measurement of electron antineutrino oscillation amplitude and frequency at Daya Bay, Phys. Rev. Lett. 112 (2014) 061801 [arXiv: 1310.6732] [INSPIRE].

[3] Daya Bay collaboration, C. Zhang, Recent results from the Daya Bay experiment, talk given at the Neutrino 2014 Conference, Boston U.S.A. (2014) [arXiv:1501.04991] [INSPIRE].

[4] RENO collaboration, J.K. Ahn et al., Observation of reactor electron antineutrino disappearance in the RENO experiment, Phys. Rev. Lett. 108 (2012) 191802 [arXiv: 1204.0626] [INSPIRE].

[5] D.V. Forero, M. Tortola and J.W.F. Valle, Neutrino oscillations refitted, Phys. Rev. D 90 (2014) 093006 [arXiv: 1405.7540] [inSPIRE].

[6] F. Capozzi et al., Status of three-neutrino oscillation parameters, circa 2013, Phys. Rev. D 89 (2014) 093018 [arXiv:1312.2878] [INSPIRE].

[7] M.C. Gonzalez-Garcia, M. Maltoni and T. Schwetz, Updated fit to three neutrino mixing: status of leptonic CP-violation, JHEP 11 (2014) 052 [arXiv:1409.5439] [INSPIRE].

[8] R.N. Mohapatra and A.Y. Smirnov, Neutrino mass and new physics, Ann. Rev. Nucl. Part. Sci. 56 (2006) 569 [hep-ph/0603118] [INSPIRE].

[9] S. Morisi and J.W.F. Valle, Neutrino masses and mixing: a flavour symmetry roadmap, Fortsch. Phys. 61 (2013) 466 [arXiv: 1206.6678] [INSPIRE].

[10] S.F. King, A. Merle, S. Morisi, Y. Shimizu and M. Tanimoto, Neutrino mass and mixing: from theory to experiment, New J. Phys. 16 (2014) 045018 [arXiv:1402.4271] [inSPIRE]. 
[11] Double Chooz collaboration, Y. Abe et al., Indication for the disappearance of reactor electron antineutrinos in the Double Chooz experiment, Phys. Rev. Lett. 108 (2012) 131801 [arXiv: 1112.6353] [INSPIRE].

[12] Double Chooz collaboration, Y. Abe et al., Reactor electron antineutrino disappearance in the Double Chooz experiment, Phys. Rev. D 86 (2012) 052008 [arXiv:1207.6632] [INSPIRE].

[13] MINOS collaboration, P. Adamson et al., Electron neutrino and antineutrino appearance in the full MINOS data sample, Phys. Rev. Lett. 110 (2013) 171801 [arXiv:1301.4581] [INSPIRE].

[14] T2K collaboration, K. Abe et al., Observation of electron neutrino appearance in a muon neutrino beam, Phys. Rev. Lett. 112 (2014) 061802 [arXiv:1311.4750] [INSPIRE].

[15] T2K collaboration, K. Abe et al., Evidence of electron neutrino appearance in a muon neutrino beam, Phys. Rev. D 88 (2013) 032002 [arXiv:1304.0841] [INSPIRE].

[16] H. Nunokawa, S.J. Parke and J.W.F. Valle, CP violation and neutrino oscillations, Prog. Part. Nucl. Phys. 60 (2008) 338 [arXiv:0710.0554] [INSPIRE].

[17] S. Pascoli and T. Schwetz, Prospects for neutrino oscillation physics, Adv. High Energy Phys. 2013 (2013) 503401 [inSPIRE].

[18] S.K. Agarwalla, S. Prakash and S. Uma Sankar, Exploring the three flavor effects with future superbeams using liquid argon detectors, JHEP 03 (2014) 087 [arXiv:1304.3251] [INSPIRE].

[19] S.K. Agarwalla, Physics potential of long-baseline experiments, Adv. High Energy Phys. 2014 (2014) 457803 [arXiv: 1401.4705] [INSPIRE].

[20] H. Minakata, Neutrino physics now and in the near future, arXiv:1403.3276 [INSPIRE].

[21] P. Minkowski, $\mu \rightarrow$ er at a rate of one out of $10^{9}$ muon decays?, Phys. Lett. B 67 (1977) 421 [INSPIRE].

[22] T. Yanagida, Horizontal symmetry and masses of neutrinos, Conf. Proc. C 7902131 (1979) 95 [INSPIRE].

[23] R.N. Mohapatra and G. Senjanović, Neutrino mass and spontaneous parity violation, Phys. Rev. Lett. 44 (1980) 912 [INSPIRE].

[24] M. Gell-Mann, P. Ramond and R. Slansky, Complex spinors and unified theories, Conf. Proc. C 790927 (1979) 315 [arXiv:1306.4669] [INSPIRE].

[25] J. Schechter and J.W.F. Valle, Neutrino masses in $\mathrm{SU}(2) \times \mathrm{U}(1)$ theories, Phys. Rev. D 22 (1980) 2227 [INSPIRE].

[26] G. Lazarides, Q. Shafi and C. Wetterich, Proton lifetime and fermion masses in an $\mathrm{SO}(10)$ model, Nucl. Phys. B 181 (1981) 287 [INSPIRE].

[27] R.N. Mohapatra and J.W.F. Valle, Neutrino mass and baryon number nonconservation in superstring models, Phys. Rev. D 34 (1986) 1642 [InSPIRE].

[28] E.K. Akhmedov, M. Lindner, E. Schnapka and J.W.F. Valle, Left-right symmetry breaking in NJLS approach, Phys. Lett. B 368 (1996) 270 [hep-ph/9507275] [INSPIRE].

[29] E.K. Akhmedov, M. Lindner, E. Schnapka and J.W.F. Valle, Dynamical left-right symmetry breaking, Phys. Rev. D 53 (1996) 2752 [hep-ph/9509255] [INSPIRE].

[30] P.S.B. Dev and R.N. Mohapatra, TeV scale inverse seesaw in $\mathrm{SO}(10)$ and leptonic non-unitarity effects, Phys. Rev. D 81 (2010) 013001 [arXiv:0910.3924] [INSPIRE]. 
[31] S.M. Boucenna, S. Morisi and J.W.F. Valle, The low-scale approach to neutrino masses, Adv. High Energy Phys. 2014 (2014) 831598 [arXiv:1404.3751] [INSPIRE].

[32] T.P. Cheng and L.-F. Li, Neutrino masses, mixings and oscillations in $\mathrm{SU}(2) \times \mathrm{U}(1)$ models of electroweak interactions, Phys. Rev. D 22 (1980) 2860 [inSPIRE].

[33] A. Zee, A theory of lepton number violation, neutrino Majorana mass and oscillation, Phys. Lett. B 93 (1980) 389 [Erratum ibid. B 95 (1980) 461] [INSPIRE].

[34] K.S. Babu, Model of 'calculable' Majorana neutrino masses, Phys. Lett. B 203 (1988) 132 [INSPIRE].

[35] M.A. Diaz, J.C. Romao and J.W.F. Valle, Minimal supergravity with R-parity breaking, Nucl. Phys. B 524 (1998) 23 [hep-ph/9706315] [INSPIRE].

[36] M. Hirsch, M.A. Diaz, W. Porod, J.C. Romao and J.W.F. Valle, Neutrino masses and mixings from supersymmetry with bilinear $R$ parity violation: a theory for solar and atmospheric neutrino oscillations, Phys. Rev. D 62 (2000) 113008 [Erratum ibid. D 65 (2002) 119901] [hep-ph/0004115] [INSPIRE].

[37] E. Roulet, MSW effect with flavor changing neutrino interactions, Phys. Rev. D 44 (1991) 935 [INSPIRE].

[38] M.M. Guzzo, A. Masiero and S.T. Petcov, On the MSW effect with massless neutrinos and no mixing in the vacuum, Phys. Lett. B 260 (1991) 154 [INSPIRE].

[39] V.D. Barger, R.J.N. Phillips and K. Whisnant, Solar neutrino solutions with matter enhanced flavor changing neutral current scattering, Phys. Rev. D 44 (1991) 1629 [INSPIRE].

[40] S. Bergmann, Y. Grossman and D.M. Pierce, Can lepton flavor violating interactions explain the atmospheric neutrino problem?, Phys. Rev. D 61 (2000) 053005 [hep-ph/9909390] [INSPIRE].

[41] Z. Berezhiani and A. Rossi, Limits on the nonstandard interactions of neutrinos from $e^{+} e^{-}$ colliders, Phys. Lett. B 535 (2002) 207 [hep-ph/0111137] [InSPIRE].

[42] S. Antusch, J.P. Baumann and E. Fernandez-Martinez, Non-standard neutrino interactions with matter from physics beyond the standard model, Nucl. Phys. B 810 (2009) 369 [arXiv:0807.1003] [INSPIRE].

[43] M.B. Gavela, D. Hernandez, T. Ota and W. Winter, Large gauge invariant non-standard neutrino interactions, Phys. Rev. D 79 (2009) 013007 [arXiv:0809.3451] [INSPIRE].

[44] M. Malinsky, T. Ohlsson and H. Zhang, Non-standard neutrino interactions from a triplet seesaw model, Phys. Rev. D 79 (2009) 011301 [arXiv:0811.3346] [InSPIRE].

[45] T. Ohlsson, T. Schwetz and H. Zhang, Non-standard neutrino interactions in the Zee-Babu model, Phys. Lett. B 681 (2009) 269 [arXiv:0909.0455] [INSPIRE].

[46] J. Kopp, M. Lindner, T. Ota and J. Sato, Non-standard neutrino interactions in reactor and superbeam experiments, Phys. Rev. D 77 (2008) 013007 [arXiv:0708.0152] [INSPIRE].

[47] R. Leitner, M. Malinsky, B. Roskovec and H. Zhang, Non-standard antineutrino interactions at Daya Bay, JHEP 12 (2011) 001 [arXiv:1105.5580] [InSPIRE].

[48] I. Girardi and D. Meloni, Constraining new physics scenarios in neutrino oscillations from Daya Bay data, Phys. Rev. D 90 (2014) 073011 [arXiv:1403.5507] [INSPIRE].

[49] C. Biggio, M. Blennow and E. Fernandez-Martinez, General bounds on non-standard neutrino interactions, JHEP 08 (2009) 090 [arXiv:0907.0097] [INSPIRE]. 
[50] Z. Berezhiani, R.S. Raghavan and A. Rossi, Probing nonstandard couplings of neutrinos at the Borexino detector, Nucl. Phys. B 638 (2002) 62 [hep-ph/0111138] [INSPIRE].

[51] O.G. Miranda, M.A. Tortola and J.W.F. Valle, Are solar neutrino oscillations robust?, JHEP 10 (2006) 008 [hep-ph/0406280] [INSPIRE].

[52] J. Barranco, O.G. Miranda, C.A. Moura and J.W.F. Valle, Constraining non-standard interactions in $\nu_{e}$ e or $\overline{n u}_{e}$ e scattering, Phys. Rev. D 73 (2006) 113001 [hep-ph/0512195] [INSPIRE].

[53] J. Barranco, O.G. Miranda, C.A. Moura and J.W.F. Valle, Constraining non-standard neutrino-electron interactions, Phys. Rev. D 77 (2008) 093014 [arXiv:0711.0698] [INSPIRE].

[54] A. Bolanos, O.G. Miranda, A. Palazzo, M.A. Tortola and J.W.F. Valle, Probing non-standard neutrino-electron interactions with solar and reactor neutrinos, Phys. Rev. D 79 (2009) 113012 [arXiv:0812.4417] [INSPIRE].

[55] F.J. Escrihuela, O.G. Miranda, M.A. Tortola and J.W.F. Valle, Constraining nonstandard neutrino-quark interactions with solar, reactor and accelerator data, Phys. Rev. D 80 (2009) 105009 [Erratum ibid. D 80 (2009) 129908] [arXiv:0907.2630] [INSPIRE].

[56] S. Davidson, C. Pena-Garay, N. Rius and A. Santamaria, Present and future bounds on nonstandard neutrino interactions, JHEP 03 (2003) 011 [hep-ph/0302093] [INSPIRE].

[57] F.J. Escrihuela, M. Tortola, J.W.F. Valle and O.G. Miranda, Global constraints on muon-neutrino non-standard interactions, Phys. Rev. D 83 (2011) 093002 [arXiv:1103.1366] [INSPIRE].

[58] D.V. Forero and M.M. Guzzo, Constraining nonstandard neutrino interactions with electrons, Phys. Rev. D 84 (2011) 013002 [INSPIRE].

[59] N. Fornengo, M. Maltoni, R. Tomas and J.W.F. Valle, Probing neutrino nonstandard interactions with atmospheric neutrino data, Phys. Rev. D 65 (2002) 013010 [hep-ph/0108043] [INSPIRE].

[60] M.C. Gonzalez-Garcia and M. Maltoni, Determination of matter potential from global analysis of neutrino oscillation data, JHEP 09 (2013) 152 [arXiv:1307.3092] [INSPIRE].

[61] S. Choubey and T. Ohlsson, Bounds on non-standard neutrino interactions using PINGU, Phys. Lett. B 739 (2014) 357 [arXiv:1410.0410] [INSPIRE].

[62] Y.-F. Li and Y.-L. Zhou, Shifts of neutrino oscillation parameters in reactor antineutrino experiments with non-standard interactions, Nucl. Phys. B 888 (2014) 137 [arXiv: 1408.6301] [INSPIRE].

[63] T. Ohlsson and H. Zhang, Non-standard interaction effects at reactor neutrino experiments, Phys. Lett. B 671 (2009) 99 [arXiv:0809.4835] [INSPIRE].

[64] S.M. Bilenky and C. Giunti, Seesaw type mixing and $\nu_{\mu} \rightarrow \nu_{\tau}$ oscillations, Phys. Lett. B 300 (1993) 137 [hep-ph/9211269] [INSPIRE].

[65] Y. Grossman, Nonstandard neutrino interactions and neutrino oscillation experiments, Phys. Lett. B 359 (1995) 141 [hep-ph/9507344] [INSPIRE].

[66] M.C. Gonzalez-Garcia, Y. Grossman, A. Gusso and Y. Nir, New CP-violation in neutrino oscillations, Phys. Rev. D 64 (2001) 096006 [hep-ph/0105159] [INSPIRE]. 
[67] D. Meloni, T. Ohlsson, W. Winter and H. Zhang, Non-standard interactions versus non-unitary lepton flavor mixing at a neutrino factory, JHEP 04 (2010) 041 [arXiv: 0912.2735] [INSPIRE].

[68] S. Antusch, C. Biggio, E. Fernandez-Martinez, M.B. Gavela and J. Lopez-Pavon, Unitarity of the leptonic mixing matrix, JHEP 10 (2006) 084 [hep-ph/0607020] [INSPIRE].

[69] S.K. Agarwalla, D.V. Forero and M. Tórtola, Spectral analysis of Daya Bay data to investigate non-standard interactions, work in progress, (2015).

[70] P. Langacker and D. London, Lepton number violation and massless nonorthogonal neutrinos, Phys. Rev. D 38 (1988) 907 [INSPIRE].

[71] D.V. Forero, M. Tortola and J.W.F. Valle, Global status of neutrino oscillation parameters after Neutrino-2012, Phys. Rev. D 86 (2012) 073012 [arXiv: 1205.4018] [InSPIRE].

[72] E. Fernandez-Martinez, M.B. Gavela, J. Lopez-Pavon and O. Yasuda, CP-violation from non-unitary leptonic mixing, Phys. Lett. B 649 (2007) 427 [hep-ph/0703098] [INSPIRE].

[73] S. Goswami and T. Ota, Testing non-unitarity of neutrino mixing matrices at neutrino factories, Phys. Rev. D 78 (2008) 033012 [arXiv:0802.1434] [INSPIRE].

[74] S. Luo, Non-unitary deviation from the tri-bimaximal lepton mixing and its implications on neutrino oscillations, Phys. Rev. D 78 (2008) 016006 [arXiv:0804.4897] [INSPIRE].

[75] D.V. Forero, S. Morisi, M. Tortola and J.W.F. Valle, Lepton flavor violation and non-unitary lepton mixing in low-scale type-I seesaw, JHEP 09 (2011) 142 [arXiv:1107.6009] [INSPIRE].

[76] V. Kopeikin, L. Mikaelyan and V. Sinev, Reactor as a source of antineutrinos: thermal fission energy, Phys. Atom. Nucl. 67 (2004) 1892 [Yad. Fiz. 67 (2004) 1916] [hep-ph/0410100] [INSPIRE].

[77] T. Mueller et al., Improved predictions of reactor antineutrino spectra, Phys. Rev. C 83 (2011) 054615 [arXiv:1101.2663] [INSPIRE].

[78] K.N. Abazajian et al., Light sterile neutrinos: a white paper, arXiv:1204.5379 [INSPIRE].

[79] P. Vogel and J.F. Beacom, Angular distribution of neutron inverse beta decay, $\bar{\nu}_{e}+p \rightarrow e^{+}+n$, Phys. Rev. D 60 (1999) 053003 [hep-ph/9903554] [INSPIRE].

[80] R. Adhikari, S. Chakraborty, A. Dasgupta and S. Roy, Non-standard interaction in neutrino oscillations and recent Daya Bay, T2K experiments, Phys. Rev. D 86 (2012) 073010 [arXiv: 1201.3047] [INSPIRE].

[81] I. Girardi, D. Meloni and S.T. Petcov, The Daya Bay and T2K results on $\sin ^{2} 2 \theta_{13}$ and non-standard neutrino interactions, Nucl. Phys. B 886 (2014) 31 [arXiv:1405.0416] [INSPIRE].

[82] A. Di Iura, I. Girardi and D. Meloni, Probing new physics scenarios in accelerator and reactor neutrino experiments, J. Phys. G 42 (2015) 065003 [arXiv:1411.5330] [INSPIRE].

[83] P. Huber, On the determination of anti-neutrino spectra from nuclear reactors, Phys. Rev. C 84 (2011) 024617 [Erratum ibid. C 85 (2012) 029901] [arXiv: 1106.0687] [INSPIRE].

[84] A.C. Hayes, J.L. Friar, G.T. Garvey, G. Jungman and G. Jonkmans, Systematic uncertainties in the analysis of the reactor neutrino anomaly, Phys. Rev. Lett. 112 (2014) 202501 [arXiv: 1309.4146] [INSPIRE]. 